\title{
Sumudu Transformation or What Else Can Laplace Transformation Do
}

\author{
Alfred Wünsche \\ Max-Planck-Group "Nonclassical Radiation” at Physical Institute of Humboldt University Berlin, \\ Berlin, Germany \\ Email: alfred.wuensche@physik.hu-berlin.de
}

How to cite this paper: Wünsche, A. (2019) Sumudu Transformation or What Else Can Laplace Transformation Do. $A d$ vances in Pure Mathematics, 9, 111-142. https://doi.org/10.4236/apm.2019.92007

Received: January 2, 2019

Accepted: February 23, 2019

Published: February 26, 2019

Copyright $\odot 2019$ by author(s) and Scientific Research Publishing Inc. This work is licensed under the Creative Commons Attribution International License (CC BY 4.0).

http://creativecommons.org/licenses/by/4.0/ (c) (i) Open Access

\begin{abstract}
The transition from a known Taylor series $f(x)=\sum_{n=0}^{\infty} \frac{f^{(n)}(0)}{n !} x^{n}$ of a known function $f(x)$ to a new function $\hat{f}(x)$ primarily defined by the infinite power series $\hat{f}(x) \equiv \sum_{n=0}^{\infty} f^{(n)}(0) x^{n}$ with coefficients $f^{(n)}(0)$ from the Taylor series of the function $f(x)$ can be made by an integral transformation which is a modified Laplace transformation and is called Sumudu transformation. It makes the transition from the Exponential series to the Geometric series and may help to evaluate new infinite power series from known Taylor series. The Sumudu transformation is demonstrated to be a limiting case of Fractional integration. Apart from the basic Sumudu integral transformation we discuss a modification where the coefficients $\frac{f^{(n)}(0)}{n !}$ from the Taylor series are not changed to $f^{(n)}(0)$ but only to $\frac{f^{(n)}(0)}{\left(\frac{n}{2}\right) !}$. Be-
\end{abstract} side simple examples our applications are mainly concerned to calculate new Generating functions for Hermite polynomials from the basic ones.

\section{Keywords}

Mellin Transformation, Fractional Integration, Geometric Series and Exponential Series, Error Function, Laguerre Polynomials, Generating Functions of Hermite Polynomials, Bessel Functions, Asymptotic Series, Operator Identities

\section{Introduction}

Let us suppose that we know the Taylor series of a function $f(x)$ 


$$
f(x)=\sum_{n=0}^{\infty} \frac{f^{(n)}(0)}{n !} x^{n}, \quad\left(f^{(n)}(0) \equiv\left\{\frac{\partial^{n}}{\partial x^{n}} f(x)\right\}_{x \rightarrow 0}\right) .
$$

It is not rare that we want to evaluate a new primarily unknown function $\hat{f}(x)$ given by its power series of the following kind

$$
\hat{f}(x) \equiv \sum_{n=0}^{\infty} f^{(n)}(0) x^{n}
$$

with coefficients $f^{(n)}(0)$ taken from the Taylor series of $f(x)$ in (1.1). This can be made by a linear integral transformation of the function $f(x)$ which is closely related to a Laplace transformation and which is now also called Sumudu transformation ${ }^{1}$. It was introduced by Watugala [1] and further developed by Belgacem and coauthors, e.g., [2] [3] [4] [5] [6] and many others, e.g., [7]. The article [2] contains basic examples of Sumudu transforms of functions. Since Laplace transformations are widely applied in mathematics for the solution of ordinary differential equations which have important applications in physics (e.g., electrical engineering) and in other sciences and since there exist detailed investigations of these transformations and voluminous tables, e.g., [8] [9] [10] [11] [12] it seems to be possible to enlarge the store of power series which can be evaluated in closed form. The present article applies the Sumudu transformation mainly to obtain new Generating functions for Hermite polynomials. In the following we derive and explain this integral transformation and consider in Section 5 a modified similar transformation which may be considered in a loose sense as half the part of the way from the original function to its Sumudu transform. In Section 3 we demonstrate how Sumudu transformation can also be considered as a limiting case of Fractional integration.

Using the well-known sequence of integrals with the real parameter $x \geq 0$

$$
\int_{0}^{+\infty} \mathrm{d} y y^{n} \exp \left(-\frac{y}{x}\right)=n ! x^{n+1}, \quad(n=0,1,2, \cdots ; x=|x| \geq 0),
$$

we obtain from the Taylor series of $f(x)$ the following general identity

$$
\begin{aligned}
\hat{f}(x) & \equiv \sum_{n=0}^{\infty} f^{(n)}(0) x^{n}=\frac{1}{x} \sum_{n=0}^{\infty} \frac{f^{(n)}(0)}{n !}\left(n ! x^{n+1}\right) \\
& =\frac{1}{x} \int_{0}^{+\infty} \mathrm{d} y\left(\sum_{n=0}^{\infty} \frac{f^{(n)}(0)}{n !} y^{n}\right) \exp \left(-\frac{y}{x}\right),
\end{aligned}
$$

and, finally, if we substitute herein (1.1)

$$
\hat{f}(x)=\frac{1}{x} \int_{0}^{+\infty} \mathrm{d} y f(y) \exp \left(-\frac{y}{x}\right), \quad(x=|x|>0) .
$$

We inserted $n ! x^{n+1}$ in (1.4) according to (1.3) and changed the order of summation and integration due to supposed absolute convergence of the sum. From (1.5) it can be seen that the functions $f(x)$ have to be determined only for $x>0$. Setting $f(x)=f(x) \theta(x)$ with $\theta(x)$ the Heaviside step function ${ }^{1}$ Sumudu means "smooth, noble" in Sanscrit and in Sinhala, the language of Sinhalese people of Sri Lanka. 
$(\theta(x)=0$ for $x<0, \theta(x)=1$ for $x>0)$ in the sense of generalized functions one may extend the integration in (1.5) from $-\infty$ to $+\infty$ that makes some proofs easier, e.g., inversion of the transformation.

By the substitution of the parameter $x \equiv \frac{1}{S}$ we obtain from (1.5) the form

$$
\frac{1}{s} \hat{f}\left(\frac{1}{s}\right)=\int_{0}^{+\infty} \mathrm{d} y f(y) \mathrm{e}^{-s y} .
$$

If we denote the standard form of the Laplace transformation $f(x) \rightarrow F(s)$ by [8] [9]

$$
f(x) \rightarrow F(s) \equiv \mathcal{L}\{f\}(s) \equiv \int_{0}^{+\infty} \mathrm{d} y f(y) \mathrm{e}^{-s y},
$$

the transition $f(x) \rightarrow \hat{f}(x)$ is found to be a modified Laplace transformation in the following sense

$$
f(x) \rightarrow \hat{f}(x)=\frac{1}{x} F\left(\frac{1}{x}\right)=\frac{1}{x} \mathcal{L}\{f\}\left(\frac{1}{x}\right) \equiv \mathcal{S}\{f\}(x), \quad(x \geq 0),
$$

where $\mathcal{S}\{\ldots\}$ stands here for Sumudu transformation. To each pair of original function $f(x)$ and its Laplace transform $F(s)$ which one may calculate or can find in tables (e.g., [8] [9] [10]) one obtains a pair of Taylor series of $f(x)$ and $\hat{f}(x)$ if one of these series is known ${ }^{2}$.

By the substitution $u \equiv \frac{y}{x}$ with $x$ as positive parameter and using partial integration we can bring (1.5) to the form

$$
\hat{f}(x)=\int_{0}^{+\infty} \mathrm{d} u f(x u) \mathrm{e}^{-u}=\int_{0}^{+\infty} \mathrm{d} u \mathrm{e}^{-u}\left(x^{u} \frac{\partial}{\partial u} f(u)\right),
$$

which by partial integration leads to

$$
\hat{f}(x)=\int_{0}^{+\infty} \mathrm{d} u f(u) \underbrace{\left(x^{-\frac{\partial}{\partial u} u} \mathrm{e}^{-u}\right)}_{\frac{1}{x} \exp \left(-\frac{u}{x}\right)},
$$

that means again to the starting form (1.5). We wrote here the multiplication of the argument of a function $f(x) \rightarrow f(a x)$ by a factor $a$ in operator form as [14] $]^{3}$

$$
f(a x)=a^{x \frac{\partial}{\partial x}} f(x) .
$$

On the other side we may consider the function $f(x u)$ in (1.9) as multipli${ }^{2}$ The last cited tables [10] contain the so-called Laplace-Carson transforms $\bar{F}(p)=p \int_{0}^{+\infty} \mathrm{d} p \mathrm{e}^{-p t} f(t)$ and thus they are related with the standard Laplace transforms $F(p)$ simply by $F(p) \rightarrow \bar{F}(p)=p F(p)=p L\{f\}(p)$.

${ }^{3}$ We mention that the operators $K_{-} \equiv \frac{1}{2} \frac{\partial^{2}}{\partial x^{2}}, \quad K_{0} \equiv \frac{1}{4}\left(x \frac{\partial}{\partial x}+\frac{\partial}{\partial x} x\right)=\frac{x}{2} \frac{\partial}{\partial x}+\frac{1}{4}, \quad K_{+} \equiv \frac{1}{2} x^{2}$, with the commutation relations $\left[K_{-}, K_{+}\right]=2 K_{0},\left[K_{0}, K_{-}\right]=-K_{-},\left[K_{0}, K_{+}\right]=+K_{+}$, form a basis of a realization of the Lie algebra $s u(1,1)$ to the Lie group $S U(1,1)$ with useful applications to Hermite polynomials. 
cation of the argument $x$ of the function $f(x)$ by a factor $u$ and using then again (1.11) we can represent (1.9) in the form

$$
\begin{aligned}
\hat{f}(x) & =\int_{0}^{+\infty} \mathrm{d} u \mathrm{e}^{-u} u^{x \frac{\partial}{\partial x}} f(x)=\underbrace{\int_{0}^{+\infty} \mathrm{d} u \mathrm{e}^{-u} u^{\frac{\partial}{\partial x} x-1}}_{\text {Mellin tr. of } \mathrm{e}^{-u}} f(x) \\
& =\Gamma\left(\frac{\partial}{\partial x} x\right) f(x) \equiv\left(x \frac{\partial}{\partial x}\right) ! f(x) .
\end{aligned}
$$

The operator which transforms $f(x)$ into $\hat{f}(x)$ and which has to be applied to $f(x)$ is here fully separated from $f(x)$ and represents the Mellin transformation [9] (chap. VI) of the function $\mathrm{e}^{-u}$ with argument substituted by the operator $\frac{\partial}{\partial x} x$. The Mellin transform $\mathcal{M}\{f\}(s)$ of a function $f(u)$ is defined by

$$
\mathcal{M}\{f\}(s) \equiv \int_{0}^{+\infty} \mathrm{d} u f(u) u^{s-1},
$$

and its special case of the function $f(u)=\mathrm{e}^{-u}$ leads to

$$
\mathcal{M}\left\{\mathrm{e}^{-u}\right\}(s)=\int_{0}^{+\infty} \mathrm{d} u \mathrm{e}^{-u} u^{s-1}=\Gamma(s) \equiv(s-1) !,
$$

and may be considered as one of the possible basic definitions of the Gamma function. Extensive tables of Mellin transforms of functions we find in cited work of Bateman and Erdélyi [9]. Beside more insight into the nature of Sumudu transformations we hope that the representation (1.12) can be applied in future also more directly using the many known formulae for the Gamma function.

We now consider the inversion of the transformation

$$
f(x) \rightarrow \hat{f}(x)=\int_{0}^{+\infty} \mathrm{d} u f(x u) \mathrm{e}^{-u} .
$$

As it is known the inversion of the Laplace transformation (1.7) is (e.g., [8] [9])

$$
F(s) \rightarrow f(x)=\mathcal{L}^{-1}\{F\}(x)=\frac{1}{\mathrm{i} 2 \pi} \int_{c-\mathrm{i} \infty}^{c+\mathrm{i} \infty} \mathrm{d} s F(s) \mathrm{e}^{x s},
$$

where real $c$ is widely arbitrary but must be chosen in the region where the integral is convergent. For the Sumudu transformation (1.5) written in the form (1.6) as a Laplace transformation of $f(x)$ this leads to the inversion

$$
f(x)=\frac{1}{\mathrm{i} 2 \pi} \int_{c-\mathrm{i} \infty}^{c+\mathrm{i} \infty} \mathrm{d} s \frac{1}{s} \hat{f}\left(\frac{1}{s}\right) \mathrm{e}^{x s} .
$$

This transformation allows to evaluate the Taylor series of a function $f(x)$ from the known function $\hat{f}(x)$ if the integral (1.17) can be evaluated.

As a first example which gives a deeper insight into the nature of the Sumudu transformation we consider the functions $f(x)=x^{n}$. Using (1.5) together with (1.3) one calculates that these functions are transformed into functions $\hat{f}(x)$ according to

$$
f(x)=x^{n} \stackrel{\text { Sumudu }}{\longrightarrow} \hat{f}(x)=n ! x^{n}, \quad(n=0,1,2, \cdots),
$$

that due to linearity of the transformation can be immediately applied to the 
Sumudu transformation of Taylor series. This correspondence possesses yet another aspect. For this purpose we mention that $x^{n}$ is eigenfunction of the operator $\frac{\partial}{\partial x} x$ to eigenvalue $n+1$ and we conclude

$$
\left(\frac{\partial}{\partial x} x\right) x^{n}=(n+1) x^{n}, \Leftrightarrow \Gamma\left(\frac{\partial}{\partial x} x\right) x^{n}=\Gamma(n+1) x^{n}=n ! x^{n},
$$

and from this (existence of Taylor series assumed)

$$
\Gamma\left(\frac{\partial}{\partial x} x\right) f(x)=\sum_{n=0}^{\infty} \frac{f^{(n)}(0)}{n !} \Gamma\left(\frac{\partial}{\partial x} x\right) x^{n}=\sum_{n=0}^{\infty} f^{(n)}(0) x^{n} \equiv \hat{f}(x) .
$$

The second part of (1.19) follows immediately from the representation (1.12) of the Sumudu transformation. However, this representation (1.12) alone is not very useful for summing up Taylor series as result of the transformation if we do not possess in addition integral representations of the form (1.5) which allow, at least in principle, to sum up the arising series by calculating an integral.

As generalization one may consider a transformation $F(x) \rightarrow \hat{F}(x)$ of the kind

$$
\hat{F}(x)=G\left(x \frac{\partial}{\partial x}\right) F(x),
$$

where $G(u)$ is an arbitrary given function and $F(x)$ the function to transform. Since $x^{n}$ are eigenfunctions of the operator $x \frac{\partial}{\partial x}$ to eigenvalue $n$ from (1.20) follows for $F(x)=x^{n}$

$$
\left(x \frac{\partial}{\partial x}\right) x^{n}=n x^{n}, \Leftrightarrow \hat{F}(x)=G\left(x \frac{\partial}{\partial x}\right) x^{n}=G(n) x^{n},
$$

and consequently

$$
\hat{F}(x)=\sum_{n=0}^{\infty} \frac{f^{(n)}(0)}{n !} G\left(x \frac{\partial}{\partial x}\right) x^{n}=\sum_{n=0}^{\infty} f^{(n)}(0) \frac{G(n)}{n !} x^{n} .
$$

For the evaluation of arising new Taylor series after the transformation it is necessary to possess in addition to (1.21) an integral representation (or an equivalent of it) which allows to calculate more directly $\hat{F}(x)$ from given $F(x)$. In particular, this is also true for the form (1.12) of the Sumudu transformation. In this case as mentioned we have it in the basic form (1.5) of the Sumudu transformation.

\section{Some General Rules for Sumudu Transformation}

In this Section we derive and discuss some general rules for Sumudu transformations according to (1.1) and (1.2) with their explicit form given in (1.5) and denote the correspondence between $f(x)$ and $\hat{f}(x)$ by

$$
f(x) \stackrel{\text { Sumudu }}{\longrightarrow} \hat{f}(x) \text {. }
$$

Then the correspondence of a function $f(a ; x) \equiv f(a x)$ with stretching pa- 
rameter $a$ of the argument to its Sumudu transform according to

$$
f(a ; x) \equiv f(a x) \stackrel{\text { Sumudu }}{\longrightarrow} \hat{f}(a ; x)=\hat{f}(a x),
$$

is determined by

$$
\begin{aligned}
\hat{f}(a ; x) & =\frac{1}{x} \int_{0}^{+\infty} \mathrm{d} y \underbrace{f(a ; y)}_{=f(a y)} \exp \left(-\frac{y}{x}\right) \\
& =\frac{1}{a x} \int_{0}^{+\infty} \mathrm{d} z f(z) \exp \left(-\frac{z}{a x}\right)=\hat{f}(a x),
\end{aligned}
$$

where $\hat{f}(x)$ denotes the Sumudu transform of $f(x)$ according to (2.1). Thus the multiplication of the argument of a function by a number a leads to a simple relation of its Sumudu transform to the Sumudu transform of the primary function and it is not necessary to consider separately Sumudu transformations of functions $f(a x)$. For the case of an argument translation of a function this is a little more complicated (see below).

To the differentiation $f^{(1)}(x) \equiv \frac{\partial}{\partial x} f(x) \equiv f_{(-1)}(x)$ of a function corresponds a function $\hat{f}_{(-1)}(x)$ using partial integration according to

$$
\begin{aligned}
\hat{f}_{(-1)}(x) & =\frac{1}{x} \int_{0}^{+\infty} \mathrm{d} y\left(\frac{\partial}{\partial y} f(y)\right) \exp \left(-\frac{y}{x}\right) \\
& =\frac{1}{x}\left\{f(y) \exp \left(-\frac{y}{x}\right)\right\}_{y=0}^{y \rightarrow+\infty}-\frac{1}{x} \int_{0}^{+\infty} \mathrm{d} y f(y)\left(\frac{\partial}{\partial y} \exp \left(-\frac{y}{x}\right)\right),
\end{aligned}
$$

with the result of this correspondence

$$
f_{(-1)}(x) \equiv f^{(1)}(x) \stackrel{\text { Sumudu }}{\longrightarrow} \hat{f}_{(-1)}(x)=\frac{1}{x}(\hat{f}(x)-f(0)),
$$

if the upper boundary $\lim _{y \rightarrow+\infty} f(y) \exp \left(-\frac{y}{x}\right)$ does not give a contribution. In analogous way one finds after a few calculations for low numbers of $n$ the general formula $(n=1,2, \cdots)$

$$
f_{(-n)}(x) \equiv f^{(n)}(x) \stackrel{\text { Sumudu }}{\longrightarrow} \hat{f}_{(-n)}(x)=\frac{1}{x^{n}}\left(\hat{f}(x)-\sum_{k=0}^{n-1} x^{k} f^{(k)}(0)\right) .
$$

It is not difficult to prove it by complete induction.

For the displacement of the argument of a function $f(x)$ by $x_{0}$ as a parameter

$$
f\left(x_{0} ; x\right) \equiv f\left(x-x_{0}\right)=\exp \left(-x_{0} \frac{\partial}{\partial x}\right) f(x) .
$$

we find with intermediate substitution $u=y-x_{0}$ of the integration variable for the correspondence to the Sumudu transform

$$
\begin{aligned}
& f\left(x_{0} ; x\right) \stackrel{\text { Sumudu }}{\longrightarrow} \hat{f}\left(x_{0} ; x\right)=\frac{1}{x} \int_{0}^{+\infty} \mathrm{d} y f\left(y-x_{0}\right) \exp \left(-\frac{y}{x}\right) \\
& =\exp \left(-\frac{x_{0}}{x}\right) \hat{f}(x)+\frac{1}{x} \int_{0}^{x_{0}} \mathrm{~d} y f\left(y-x_{0}\right) \exp \left(-\frac{y}{x}\right) .
\end{aligned}
$$


The second sum term is some kind of incomplete Sumudu transformation of the function $f\left(x-x_{0}\right)$ and it vanishes for $x_{0}=0$. By the substitution $y-x_{0}=z$ this can be transformed to

$$
f\left(x_{0} ; x\right) \stackrel{\text { Sumudu }}{\longrightarrow} \hat{f}\left(x_{0} ; x\right)=\exp \left(-\frac{x_{0}}{x}\right) \int_{-x_{0}}^{\infty} \mathrm{d} z f(z) \exp \left(-\frac{z}{x}\right) .
$$

We mention that for even functions $f_{+}(x)=+f_{+}(-x)$ with existing Taylor series we find the correspondence

$$
\begin{aligned}
\hat{f}_{+}(x) & =\sum_{m=0}^{\infty} f_{+}^{(2 m)}(0) x^{2 m} \\
& =\frac{1}{2 x} \int_{0}^{+\infty} \mathrm{d} y(f(y)+f(-y)) \exp \left(-\frac{y}{x}\right) \\
& =\frac{1}{2|x|} \int_{-\infty}^{+\infty} \mathrm{d} y f(y) \exp \left(-\frac{y}{|x|}\right)=+\hat{f}_{+}(-x),
\end{aligned}
$$

and for odd functions $f_{-}(x)=-f_{-}(-x)$ the correspondence

$$
\begin{aligned}
\hat{f}_{-}(x) & =\sum_{m=0}^{\infty} f_{-}^{(2 m+1)}(0) x^{2 m+1} \\
& =\frac{1}{2 x} \int_{0}^{+\infty} \mathrm{d} y(f(y)-f(-y)) \exp \left(-\frac{y}{x}\right) \\
& =\frac{1}{2|x|} \int_{-\infty}^{+\infty} \mathrm{d} y \operatorname{sign}(y) f(y) \exp \left(-\frac{y}{|x|}\right)=-\hat{f}_{-}(-x)
\end{aligned}
$$

Sumudu transformation preserves the lateral symmetry of a function.

\section{Fractional Integration and Sumudu Transformation as a Certain Limiting Case}

We now consider fractional integration and differentiation (e.g., Bateman and Erdélyi [15], Vladimirov [16], Oldham and Spanier [17], Samko, Kilbas, Marichev [18]) which is often useful in connection with Sumudu transformation. Furthermore, Sumudu transformation can be represented as a certain limiting case of fractional integration as we will show in this Section. First, we shortly introduce fractional integration.

It is known (in an elementary form, e.g., Kranzer [19], p. 205) that the $n$-fold integration of a function $f(x)$ can be represented by a convolution integral as follows

$$
\begin{aligned}
f_{(n)}(x) & \equiv \int_{0}^{x} \mathrm{~d} x_{n-1} \int_{0}^{x_{n-1}} \mathrm{~d} x_{n-2} \ldots \int_{0}^{x_{2}} \mathrm{~d} x_{1} \int_{0}^{x_{1}} \mathrm{~d} x_{0} f\left(x_{0}\right) \\
& =\frac{1}{(n-1) !} \int_{0}^{x} \mathrm{~d} y(x-y)^{n-1} f(y), \quad(n=1,2, \cdots)
\end{aligned}
$$

This can be proved by complete induction first changing the order of integration in the arising double integral and then calculating the inner integral

$$
f_{(n+1)}(x)=\int_{0}^{x} \mathrm{~d} y f_{(n)}(y)=\int_{0}^{x} \mathrm{~d} y \frac{1}{(n-1) !} \int_{0}^{y} \mathrm{~d} z(y-z)^{n-1} f(z)
$$




$$
\begin{aligned}
& =\int_{0}^{x} \mathrm{~d} z f(z) \int_{z}^{x} \mathrm{~d} y \frac{(y-z)^{n-1}}{(n-1) !}=\int_{0}^{z} \mathrm{~d} z f(z)\left\{\frac{(y-z)^{n}}{n !}\right\}_{y=z}^{y=x} \\
& =\frac{1}{n !} \int_{0}^{x} \mathrm{~d} z(x-z)^{n} f(z),
\end{aligned}
$$

that together with the correct $f_{(1)}(x)$ as initial member proves the proposition.

The $n$-fold integral (3.1) can be generalized in the rank of a definition to a $v$-fold fractional integral with arbitrary real parameter $v$ by the extension $n \rightarrow v$ in (3.1) (positive real solution is to insert for the multi-valued function $z^{v-1}$ )

$$
f_{(v)}(x) \equiv \frac{1}{(v-1) !} \int_{0}^{x} \mathrm{~d} y(x-y)^{v-1} f(y)=\frac{1}{(v-1) !} \int_{0}^{x} \mathrm{~d} z z^{v-1} f(x-z) .
$$

This form is also called the Riemann-Liouville integral [15]. The case $v=0$ as limiting case provides

$$
\begin{aligned}
f_{(0)}(x) & \equiv \lim _{\varepsilon \rightarrow+0} f_{(\varepsilon)}(x)=\lim _{\varepsilon \rightarrow 0} \frac{1}{(\varepsilon-1) !} \int_{0}^{x} \mathrm{~d} z z^{\varepsilon-1} f(x-z) \\
& =\lim _{\varepsilon \rightarrow 0} \frac{\varepsilon}{\varepsilon !} \int_{0}^{x} \mathrm{~d} z z^{\varepsilon-1}\left(f(x)-\frac{z}{1 !} f^{(1)}(x)+\cdots\right) \\
& =f(x) \lim _{\varepsilon \rightarrow 0} \frac{x^{\varepsilon}-0^{\varepsilon}}{\varepsilon !}-\frac{f^{(1)}(x)}{1 !} \lim _{\varepsilon \rightarrow 0} \varepsilon \frac{z^{\varepsilon+1}-0^{\varepsilon+1}}{(\varepsilon+1) !}+\cdots=f(x) .
\end{aligned}
$$

The convolution (3.3) of $f(x)$ with powers of $x$ is one of the possible basic definitions of fractional integration of order $v>0$ of a function $f(x)$, e.g., [15] [16] [17] [18] (the last two [17] and [18] with a short historical introduction). In [15] (chap. XIII) and in [18] (pp. 140-142) one finds tables of fractional integrals. Fractional derivatives $(v<0)$ can be obtained from appropriate fractional integrals $(v \geq 0)$ by corresponding integer differentiations. Interesting is the view from the theory of the convolution algebra of generalized functions where one can introduce the functions $\theta_{(v)}(x)$ which generalize the step function [16] (p. 142; he uses $f_{v}$ instead of our $\delta_{v}$ ) by

$$
\begin{gathered}
\delta_{v}(x)=\left\{\begin{array}{ll}
\theta(x) \frac{x^{\nu-1}}{(v-1) !}, & (v>0) \\
\delta_{v+1}^{(1)}, & (v \leq 0)
\end{array} \Rightarrow \delta_{\mu}(x) * \delta_{v}(x)=\delta_{\mu+\nu}(x),\right. \\
\delta_{0}(x)=\delta(x), \quad \delta(x)=\theta(x), \quad \delta_{-n}(x)=\delta^{(n)}(x),
\end{gathered}
$$

and where “*” stands for the convolution $f(x) * g(x) \equiv \int_{-\infty}^{+\infty} \mathrm{d} y f(x-y) g(y)$. Fractional integration of functions $f_{0}(x)=f(x) \theta(x)$ can be then determined by

$$
f_{v}(x)=\delta_{v}(x) * f(x),
$$

with the consequence

$$
\delta_{\mu}(x) * f_{v}(x)=\delta_{\mu}(x) * \delta_{v}(x) * f(x)=\delta_{\mu+v}(x) * f(x)=f_{\mu+v}(x),
$$

that means associativity as it has to be for successive convolutions using a notation of the form of multiplications. 
Sumudu transformation and fractional integration of order $v$ do not commute. If we make first the fractional integration of order $v$ of the powers $x^{n},(n=0,1,2, \cdots)$ with symbol $\stackrel{\text { Fract }(v)}{\longrightarrow}$ and then with the result the Sumudu transformation we obtain

$$
x^{n} \stackrel{\text { Fract }(v)}{\longrightarrow} \frac{n !}{(n+v) !} x^{n+v} \stackrel{\text { Sumudu }}{\longrightarrow} n ! x^{n+v},
$$

and if we make first a Sumudu transformation of the powers $x^{n}$ and then a fractional integration of order $v$ of the result we find

$$
x^{n} \stackrel{\text { Sumudu }}{\longrightarrow} n ! x^{n} \stackrel{\text { Fract }(v)}{\longrightarrow} \frac{n !^{2}}{(n+v) !} x^{n+v} .
$$

In comparison, two successive fractional integrations of order $\mu$ and $v$ are commuting and result in a fractional integration of order $\mu+v$ and, therefore, form a one-parameter group with the identity operation belonging to $v=0$.

By comparison of the Laplace transforms in the tables of Bateman and Erdélyi [9] (pp. 120-204, here, in particular, p. 121, (8) and (9)) with the Sumudu transforms (2.6) one finds that essentially the variable of the Laplace transform is substituted by its inverse in the Sumudu transform. In case of negative values $v=-|v|$ we have in front of $\hat{f}(x)$ the powers $\frac{1}{|x|^{v}}$ which are singular functions of $x$ at $x=0$ where the singularities in the sense of generalized functions have to be determined more precisely.

We now consider a certain limiting transition from fractional integration to Sumudu transformation and write for this purpose (3.2) first in the way

$$
f_{(n+1)}(z)=\frac{1}{n !} \int_{0}^{z} \mathrm{~d} y(z-y)^{n} f(y)=\frac{z^{n}}{n !} \int_{0}^{z} \mathrm{~d} y\left(1-\frac{y}{z}\right)^{n} f(y),
$$

and substitute $z=n x$ with $x>0$ that leads to the following relation

$$
\frac{n !}{n^{n} x^{n+1}} f_{(n+1)}(n x)=\frac{1}{x} \int_{0}^{n x} \mathrm{~d} y\left(1-\frac{y}{n x}\right)^{n} f(y) \text {. }
$$

If we now make the limiting transition $n \rightarrow+\infty$ using the definition of the exponential functions $\mathrm{e}^{z}$ by the limiting process

$$
\lim _{n \rightarrow \infty}\left(1+\frac{z}{n}\right)^{n}=\mathrm{e}^{z}
$$

and known already to Euler we obtain from the right-hand side of (3.11) and in agreement with (1.5)

$$
\lim _{n \rightarrow \infty} \frac{1}{x} \int_{0}^{n x} \mathrm{~d} y\left(1-\frac{y}{n x}\right)^{n} f(y)=\frac{1}{x} \int_{0}^{+\infty} \mathrm{d} y \exp \left(-\frac{y}{x}\right) f(y) \equiv \hat{f}(x) .
$$

From the left-hand side of (3.11) in the limiting transition $n \rightarrow \infty$ using the well-known Stirling approximation of the Gamma function for $z \rightarrow+\infty$

$$
\Gamma(z)=\sqrt{2 \pi} \mathrm{e}^{-z} z^{z-\frac{1}{2}}\left\{1+\frac{1}{12 z}+\cdots\right\}=(z-1) !
$$


inserting this for $(n-1)$ ! we find

$$
\lim _{n \rightarrow \infty} \frac{n !}{n^{n} x^{n+1}} f_{(n+1)}(n x)=\lim _{n \rightarrow \infty} \sqrt{\frac{2 \pi n}{x^{2}}} \frac{f_{(n+1)}(n x)}{(\mathrm{e} x)^{n}}, \quad(x=|x|>0) .
$$

Thus we may write the Sumudu transformation $\hat{f}(x)$ as the following limit of the fractional integration if we substitute in addition $n \rightarrow v \in \mathbb{R}$

$$
\hat{f}(x)=\sqrt{\frac{2 \pi}{x^{2}}} \lim _{v \rightarrow \infty} \frac{\sqrt{v} f_{(v+1)}(v x)}{(\mathrm{e} x)^{v}}, \quad(x=|x|>0) .
$$

The right-hand side of this relation is one of the ways to represent the Sumu$\mathrm{du}$ transformation by a limiting procedure from fractional integration.

\section{Examples for Sumudu Transformations}

We provide now a few simple examples for Sumudu transformations. The transformation of the "basic" monomial functions $f(x)=x^{n}$ was already considered in (1.18) and (1.19) and leads to $\hat{f}(x)=n ! x^{n}$. It illustrates the action of the operator $\Gamma\left(\frac{\partial}{\partial x} x\right)$ in (1.12) in ideal way.

The most striking genuine example for a Sumudu transformation establishes the connection between the Exponential and the Geometric series, clearly, with a well-known result. One finds [2]

$$
\begin{gathered}
f(x)=\sum_{n=0}^{\infty} \frac{(-x)^{n}}{n !}=\mathrm{e}^{-x}, \quad F(t)=\frac{1}{1+t}, \\
\hat{f}(x)=\sum_{n=0}^{\infty}(-x)^{n}=\frac{1}{x} F\left(\frac{1}{x}\right)=\frac{1}{x} \int_{0}^{+\infty} \mathrm{d} y \mathrm{e}^{-y} \exp \left(-\frac{y}{x}\right)=\frac{1}{1+x} .
\end{gathered}
$$

For this example inserting $\hat{f}(x)=\frac{1}{1+x}$ on the right-hand side of the formula (1.17) it is easy to demonstrate the inverse Sumudu transformation from the Geometric to the Exponential series according to

$$
f(x)=\frac{1}{\mathrm{i} 2 \pi} \int_{c-\mathrm{i} \infty}^{c+\mathrm{i} \infty} \mathrm{d} s \frac{1}{s} \frac{1}{1+\frac{1}{S}} \mathrm{e}^{x s}=\frac{1}{\mathrm{i} 2 \pi} \int_{C} \mathrm{~d} s \frac{\mathrm{e}^{x s}}{s+1}=\mathrm{e}^{-x} .
$$

Here we applied the residue theorem for the Cauchy integral over a closed contour $C$ in the complex $s$-plane including the only singularity of the integrand at $s=-1$. The contour $C$ consists for parameter $x>0$ of the line through $s=c$ parallel to the imaginary axis closed by a semicircle $s=c+r \mathrm{e}^{\mathrm{i} \varphi}$ with $\frac{\pi}{2} \leq \varphi \leq \frac{3 \pi}{2}$ or $\cos (\varphi) \leq 0 \quad$ (i.e. to the left of the line) and followed by $r \rightarrow \infty$ where the integral over the semicircle then vanishes. The possible real values $c$ in the integral are then only restricted by $c>-1$ for the purpose to include the singularity.

A further interesting simple example establishes a connection between the Bessel function $\mathrm{J}_{0}(2 \sqrt{x})$ and the Exponential function $\mathrm{e}^{-x}$ in the following 
way [2]

$$
\begin{gathered}
f(x)=\sum_{n=0}^{\infty} \frac{(-x)^{n}}{n !^{2}}=\mathrm{J}_{0}(2 \sqrt{x}), \quad F(t)=\frac{1}{t} \exp \left(-\frac{1}{t}\right), \\
\hat{f}(x)=\sum_{n=0}^{\infty} \frac{(-x)^{n}}{n !}=\frac{1}{x} F\left(\frac{1}{x}\right)=\frac{1}{x} \int_{0}^{+\infty} \mathrm{d} y \mathrm{~J}_{0}(2 \sqrt{y}) \exp \left(-\frac{y}{x}\right)=\mathrm{e}^{-x} .
\end{gathered}
$$

In this example it is merely the integral transform from the Bessel function $\mathrm{J}_{0}(2 \sqrt{x})$ to the exponential function $\mathrm{e}^{-x}$ which is less known (but not unknown) in comparison to the other results. Clearly, by substitution $x \rightarrow \mathrm{i} x$ one may obtain similar formulae for the Bessel function $\mathrm{I}_{0}(2 \sqrt{x})$.

Related to (4.1) one finds

$$
\begin{gathered}
f(x)=\sum_{n=0}^{\infty} \frac{(-x)^{n}}{n !(n+1)}=\frac{1}{x} \int_{0}^{x} \mathrm{~d} t \mathrm{e}^{-t}=\frac{1-\mathrm{e}^{-x}}{x}, \\
\hat{f}(x)=\sum_{n=0}^{\infty} \frac{(-x)^{n}}{n+1}=\frac{1}{x} \int_{0}^{+\infty} \mathrm{d} y \frac{1-\mathrm{e}^{-y}}{y} \exp \left(-\frac{y}{x}\right)=\frac{\log (1+x)}{x},
\end{gathered}
$$

and related to $(4.3)$

$$
\begin{gathered}
f(x)=\sum_{n=0}^{\infty} \frac{(-x)^{n}}{n !(n+\mu) !}=\frac{\mathrm{J}_{\mu}(2 \sqrt{x})}{(\sqrt{x})^{\mu}} \\
\hat{f}(x)=\sum_{n=0}^{\infty} \frac{(-x)^{n}}{(n+\mu) !}=\frac{1}{x} F\left(\frac{1}{x}\right)=\frac{1}{x} \int_{0}^{+\infty} \mathrm{d} y \frac{\mathrm{J}_{\mu}(2 \sqrt{y})}{(\sqrt{y})^{\mu}} \exp \left(-\frac{y}{x}\right) .
\end{gathered}
$$

For integer $\mu=m=(0,1,2, \cdots)$ one finds from this relation

$$
\begin{aligned}
& \frac{1}{x} \int_{0}^{+\infty} \mathrm{d} y \frac{\mathrm{J}_{m}(2 \sqrt{y})}{(\sqrt{y})^{m}} \exp \left(-\frac{y}{x}\right) \\
& =\sum_{n=0}^{\infty} \frac{(-x)^{n}}{(n+m) !}=\sum_{k=m}^{\infty} \frac{(-x)^{k-m}}{k !}=\frac{(-1)^{m}}{x^{m}}\left(\exp (-x)-\sum_{k=0}^{m-1} \frac{(-x)^{k}}{k !}\right)
\end{aligned}
$$

It is here again the integral over modified Bessel functions multiplied with an exponential function which is less known than the involved Taylor series. We mention that the $n$-th derivative of the Bessel function $\mathrm{J}_{0}(2 \sqrt{x})$ leads to the entire function $(-1)^{n} \frac{\mathrm{J}_{n}(2 \sqrt{x})}{(\sqrt{x})^{n}}$ according to the more general formulae

$$
\frac{\partial^{n}}{\partial u^{n}} \frac{\mathrm{J}_{\mu}(2 \sqrt{u})}{(\sqrt{u})^{\mu}}=(-1)^{n} \frac{\mathrm{J}_{\mu+n}(2 \sqrt{u})}{(\sqrt{u})^{\mu+n}}, \quad \frac{\partial^{n}}{\partial u^{n}} \frac{\mathrm{I}_{\mu}(2 \sqrt{u})}{(\sqrt{u})^{\mu}}=\frac{\mathrm{I}_{\mu+n}(2 \sqrt{u})}{(\sqrt{u})^{\mu+n}} .
$$

This can be generalized by fractional differentiation from integer $n$ to arbitrary positive real $v$. The corresponding more general formulae for fractional integration of order $v$ are [15] (chap. 13.1. formula (63) in the table) or [18] (p. 142, Eq. 9) 


$$
\frac{1}{(v-1) !} \int_{0}^{x} \mathrm{~d} y(x-y)^{v-1}\left\{\begin{array}{l}
(\sqrt{y})^{\mu} \mathrm{J}_{\mu}(2 \sqrt{y}) \\
(\sqrt{y})^{\mu} \mathrm{I}_{\mu}(2 \sqrt{y})
\end{array}\right\}=\left\{\begin{array}{l}
(\sqrt{x})^{\mu+v} \mathrm{~J}_{\mu+v}(2 \sqrt{x}) \\
(\sqrt{x})^{\mu+v} \mathrm{I}_{\mu+v}(2 \sqrt{x})
\end{array}\right\} .
$$

The integrals (1.3) cannot be extended from positive to negative $n$ since $(-n) ! \rightarrow \infty,(n=1,2, \cdots)$. Nevertheless, the Sumudu transform can be calculated for some functions without a Taylor series at $x=0$ and it is interesting to see in an example what happens. For this purpose we calculate the Sumudu transform of the function $f(x)=\theta\left(x-x_{0}\right)$ where $\theta(x)$ is the Heaviside step function $(\theta(x)=0,(x<0), \theta(x)=1,(x>0))$. We find

$$
\begin{gathered}
f(x)=\theta\left(x-x_{0}\right), \quad\left(x_{0}>0\right), \\
\hat{f}(x)=\frac{1}{x} \int_{0}^{+\infty} \mathrm{d} y \theta\left(y-x_{0}\right) \exp \left(-\frac{y}{x}\right)=\frac{1}{x} \int_{x_{0}}^{+\infty} \mathrm{d} y \exp \left(-\frac{y}{x}\right) \\
=\exp \left(-\frac{x_{0}}{x}\right)=\sum_{n=1}^{\infty} \frac{(-1)^{n}}{n !}\left(\frac{x_{0}}{x}\right)^{n}+1 .
\end{gathered}
$$

The Sumudu transform $\hat{f}(x)$ is singular at $x=0$ with the Laurent series written in (4.9). If we formally write the inverse Sumudu transformation $f(x)$ to $\hat{f}(x)$

$$
f(x)=\sum_{n=1}^{\infty} \frac{(-1)^{n}}{n !(-n) !}\left(\frac{x_{0}}{x}\right)^{n}+\frac{1}{0 !}
$$

adding for negative powers $n<0$ of $x$ in the denominator the factor $(-n)$ ! then for $n= \pm 1, \pm 2, \cdots$ we have $\frac{1}{n !(-n) !}=0$ and the series (4.10) becomes undetermined in the neighborhood of $x=0$ due to the singularities.

The Sumudu transformation is an interesting addition to other methods of the analysis of functions but often one possesses alternative possibilities to evaluate infinite series.

\section{A modified Sumudu or a Kind of Gauss Transformation}

In analogy to the integral (1.3) we now consider the sequence of integrals for non-negative integers $n=0,1, \cdots$ with real positive parameter $x$ (identity

$$
\begin{aligned}
& \left.\sqrt{\pi}=\left(-\frac{1}{2}\right) !\right) \\
& \quad \int_{0}^{+\infty} \mathrm{d} y y^{n} \exp \left(-\frac{y^{2}}{x^{2}}\right)=\frac{1}{2}\left(\frac{n-1}{2}\right) ! x^{n+1}=\frac{\sqrt{\pi} n !}{\left(\frac{n}{2}\right) !}\left(\frac{x}{2}\right)^{n+1}, \quad(x=|x|),
\end{aligned}
$$

which after simple substitutions is related to (1.3) and extends it practically to semi-integers $n$. We used in (5.1) the well-known formula for doubling of the argument of the Gamma function $\Gamma(z)$

$$
\Gamma(2 z+1)=\frac{2^{2 z}}{\sqrt{\pi}} \Gamma\left(z+\frac{1}{2}\right) \Gamma(z+1)=\frac{2^{2 z}}{\sqrt{\pi}}\left(z-\frac{1}{2}\right) ! z !=(2 z) !,
$$


with $z=\frac{n}{2}$. Two special cases of the sequence of integrals (5.1), first, setting $n=2 m$ leads to

$$
\int_{0}^{+\infty} \mathrm{d} y y^{2 m} \exp \left(-\frac{y^{2}}{x^{2}}\right)=\frac{1}{2}\left(m-\frac{1}{2}\right) ! x^{2 m+1}=\sqrt{\pi} \frac{(2 m) !}{m !}\left(\frac{x}{2}\right)^{2 m+1},
$$

with $x \equiv \sqrt{x^{2}}>0$ for real $x^{2}$ and, second, setting $n=2 m+1$ leads to

$$
\int_{0}^{+\infty} \mathrm{d} y y^{2 m+1} \exp \left(-\frac{y^{2}}{x^{2}}\right)=\frac{1}{2} m ! x^{2 m+2}=\sqrt{\pi} \frac{(2 m+1) !}{\left(m+\frac{1}{2}\right) !}\left(\frac{x}{2}\right)^{2 m+2} \text {. }
$$

The integral (5.1) can be used to transform a function $f(x)$ with the Taylor series

$$
f(x)=\sum_{n=0}^{\infty} \frac{f^{(n)}(0)}{n !} x^{n}
$$

in analogy to (1.5) into a function $\hat{f}^{\frac{1}{2}}(x)$ in the rank of its definition by the Taylor series as follows

$$
\begin{aligned}
\hat{f}^{\frac{1}{2}}(x) & \equiv \sum_{n=0}^{\infty} \frac{f^{(n)}(0)}{\left(\frac{n}{2}\right) !}\left(\frac{x}{2}\right)^{n}=\frac{1}{\sqrt{\pi}} \sum_{n=0}^{\infty} \frac{\left(\frac{n-1}{2}\right) !}{n !} f^{(n)}(0) x^{n} \\
& =\frac{2}{\sqrt{\pi x^{2}}} \int_{0}^{+\infty} \mathrm{d} y\left(\sum_{n=0}^{\infty} \frac{f^{(n)}(0)}{n !} y^{n}\right) \exp \left(-\frac{y^{2}}{x^{2}}\right), \quad(x>0) .
\end{aligned}
$$

This transformation can be compactly written in the integral form

$$
\hat{f}^{\frac{1}{2}}(x) \equiv \frac{2}{\sqrt{\pi x^{2}}} \int_{0}^{+\infty} \mathrm{d} y f(y) \exp \left(-\frac{y^{2}}{x^{2}}\right), \quad(x>0) .
$$

This is a certain kind of a Gauss transformation of the function $f(x)^{4}$. If we are able to find the integral on the right-hand side for a function $f(x)$ we may evaluate the infinite power series denoted by $\hat{f}^{\frac{1}{2}}(x)$ on the left-hand side.

In the same way as in case of the Sumudu transformation we now substitute $u \equiv \frac{y}{x}$ with $x>0$ as a parameter and find from (5.7)

$$
\hat{f}^{\frac{1}{2}}(x)=\frac{2}{\sqrt{\pi}} \int_{0}^{+\infty} \mathrm{d} u f(x u) \exp \left(-u^{2}\right),
$$

and apply the operator representation (1.11) to the function $f(x u)$ with $u$ as a factor to the variable $x$ of the function $f(x)$. In this way follows from (5.7)

${ }^{4}$ As Gauss transformation is sometimes denoted a transformation with displacement of the argument of the Gauss function $\exp \left(-x^{2}\right)$ according to $\mathcal{G}\{f\}(x) \equiv \int_{-\infty}^{+\infty} \mathrm{d} y \exp \left(-(x-y)^{2}\right) f(y)$ [13] (chap. 10.13 (30)). In other sources [20] (from p. 170 on) almost this same transformation $F(x) \equiv \frac{1}{\sqrt{4 \pi}} \int_{-\infty}^{+\infty} \mathrm{d} y \exp \left(-\frac{(x-y)^{2}}{4}\right) f(y)$ with the kernel $\frac{1}{\sqrt{4 \pi t}} \exp \left(-\frac{x^{2}}{4 t}\right)$ from the heat equation with choice $t=1$ is called a Weierstrass transform. 


$$
\begin{aligned}
& \hat{f}^{\frac{1}{2}}(x)=\frac{2}{\sqrt{\pi}} \underbrace{\int_{0}^{+\infty} \mathrm{d} u \exp \left(-u^{2}\right) u^{\frac{\partial}{\partial x} x-1}}_{\text {Mellin tr. of } \mathrm{e}^{-u^{2}}} f(x) \\
& =\frac{1}{\sqrt{\pi}} \int_{0}^{+\infty} \mathrm{d} v \mathrm{e}^{-v} v^{\frac{1}{2} \partial x} x-1 \quad f(x)=\frac{1}{\sqrt{\pi}} \Gamma\left(\frac{1}{2} \frac{\partial}{\partial x} x\right) f(x) \\
& =\frac{1}{\left(-\frac{1}{2}\right) !}\left(\frac{x}{2} \frac{\partial}{\partial x}-\frac{1}{2}\right) ! f(x) .
\end{aligned}
$$

In analogy to (1.12) this can be represented by a Mellin transformation of the function $\mathrm{e}^{-u^{2}}$ taking into account

$$
\mathcal{M}\left\{\mathrm{e}^{-u^{2}}\right\}(s)=\int_{0}^{+\infty} \mathrm{d} u \mathrm{e}^{-u^{2}} u^{s-1}=\frac{1}{2} \Gamma\left(\frac{s}{2}\right) \equiv \frac{1}{2}\left(\frac{s}{2}-1\right) !
$$

with the argument $s$ substituted by the operator $\frac{\partial}{\partial x} x$. This explains the upper index $\frac{1}{2}$ of our notation $\hat{f}^{\frac{1}{2}}(x)$ for the transform of $f(x)$.

If we substitute the integration variable $y^{2}=z$ in (5.7) then the function $\hat{f}^{\frac{1}{2}}(x)$ with variable substitution $x \rightarrow \sqrt{|x|}>0$ takes on a form which can be written

$$
\sqrt{\pi} \frac{\hat{f}^{\frac{1}{2}}(\sqrt{|x|})}{\sqrt{|x|}}=\frac{1}{|x|} \int_{0}^{+\infty} \mathrm{d} z \frac{f(\sqrt{z})}{\sqrt{z}} \exp \left(-\frac{z}{|x|}\right) .
$$

The right-hand side of this relation possesses the form (1.5) of a Sumudu transformation of the function $\frac{f(\sqrt{|x|})}{\sqrt{|x|}}$ with $\sqrt{|x|}>0$ leading to a new function involving the function $\hat{f}^{\frac{1}{2}}(\sqrt{|x|})$ and determined by the left-hand side of (5.11). The inversion of the transformation according to (1.17) with corresponding substitutions is

$$
\frac{\tilde{f}^{\frac{1}{2}}(\sqrt{|x|})}{\sqrt{|x|}}=\frac{\sqrt{\pi}}{\mathrm{i} 2 \pi} \int_{c-\mathrm{i} \infty}^{c+\mathrm{i} \infty} \mathrm{d} s \frac{1}{s} \frac{f^{\frac{1}{2}}\left(\frac{1}{\sqrt{s}}\right)}{\frac{1}{\sqrt{s}}} \mathrm{e}^{|x| s},
$$

or with variable substitution $\sqrt{|x|} \rightarrow x>0$ back to the original function variable

$$
f(x)=\frac{\sqrt{\pi} x}{\mathrm{i} 2 \pi} \int_{c-\mathrm{i} \infty}^{c+\mathrm{i} \infty} \mathrm{d} s \frac{1}{s} \sqrt{|s|} f^{\frac{1}{2}}\left(\frac{1}{\sqrt{s}}\right) \exp \left(x^{2} s\right), \quad(x \geq 0) .
$$

We now give a few general rules for the considered transformation. The multiplication of the argument of a function $f(x)$ by a number a leads here as in the case of Sumudu transformation (2.2) again to

$$
f(a ; x)=f(a x)
$$




$$
\hat{f}^{\frac{1}{2}}(a ; x)=\frac{2}{\sqrt{\pi x^{2}}} \int_{0}^{+\infty} \mathrm{d} y f(a y) \exp \left(-\frac{y^{2}}{x^{2}}\right)=\hat{f}^{\frac{1}{2}}(a x),
$$

where $\hat{f}^{\frac{1}{2}}(x)$ is the corresponding Gauss transformation (5.7) of the function $f(x)$.

For even functions $f_{+}(x)=+f_{+}(-x)$ or combinations of them from (5.3) follows

$$
\begin{aligned}
f_{+}(x) & \equiv \frac{1}{2}(f(x)+f(-x))=\sum_{m=0}^{\infty} \frac{f^{(2 m)}(0)}{(2 m) !} x^{2 m}, \\
\hat{f}_{+}^{\frac{1}{2}}(x) & \equiv \sum_{m=0}^{\infty} \frac{f^{(2 m)}(0)}{m !}\left(\frac{x}{2}\right)^{2 m} \\
& =\frac{1}{\sqrt{\pi x^{2}}} \int_{-\infty}^{+\infty} \mathrm{d} y f(y) \exp \left(-\frac{y^{2}}{x^{2}}\right)=+\hat{f}_{+}^{\frac{1}{2}}(-x),
\end{aligned}
$$

and for the odd case $f_{-}(x)=-f_{-}(-x)$ using (5.3)

$$
\begin{gathered}
f_{-}(x) \equiv \frac{1}{2}(f(x)-f(-x))=\sum_{m=0}^{\infty} \frac{f^{(2 m+1)}(0)}{(2 m+1) !} x^{2 m+1}, \\
\hat{f}_{-}^{\frac{1}{2}}(x) \equiv \sum_{m=0}^{\infty} \frac{f^{(2 m+1)}(0)}{\left(m+\frac{1}{2}\right) !}\left(\frac{x}{2}\right)^{2 m+1} \\
=\frac{1}{\sqrt{\pi x^{2}}} \int_{-\infty}^{+\infty} \mathrm{d} y \operatorname{sign}(y) f(y) \exp \left(-\frac{y^{2}}{x^{2}}\right)=-\hat{f}_{-}^{\frac{1}{2}}(-x) .
\end{gathered}
$$

The considered Gauss transformation preserves the lateral symmetry of a function.

\section{Examples for Modified Sumudu or Gauss Transformations}

As an example for the integral transformation (5.7) we consider the case

$$
f(x)=\exp (-x), \quad f^{(n)}(0)=(-1)^{n},
$$

which belongs to the Exponential function as the primary function. For the evaluation of the corresponding series $\hat{f}^{\frac{1}{2}}(x)$ we find then

$$
\begin{aligned}
\sum_{n=0}^{\infty} \frac{(-1)^{n}}{\left(\frac{n}{2}\right) !}\left(\frac{x}{2}\right)^{n} & =\frac{2}{\sqrt{\pi x^{2}}} \int_{0}^{+\infty} \mathrm{d} y \exp (-y) \exp \left(-\frac{y^{2}}{x^{2}}\right) \\
& =\frac{2}{\sqrt{\pi x^{2}}} \exp \left(\frac{x^{2}}{4}\right) \int_{0}^{+\infty} \mathrm{d} y \exp \left(-\frac{1}{x^{2}}\left(y+\frac{x^{2}}{2}\right)^{2}\right) \\
& =\frac{2}{\sqrt{\pi}} \exp \left(\frac{x^{2}}{4}\right)\left\{\int_{0}^{+\infty} \mathrm{d} u \exp \left(-u^{2}\right)-\int_{0}^{\frac{x}{2}} \mathrm{~d} u \exp \left(-u^{2}\right)\right\} .
\end{aligned}
$$

The integrals on the right-hand side are well-known Special functions which can be represented by the Error function $\operatorname{Erf}(z)$ in the following way (we 
substitute $\frac{x}{2} \equiv z$ )

$$
\sum_{n=0}^{\infty} \frac{(-1)^{n}}{\left(\frac{n}{2}\right) !} z^{n}=\exp \left(z^{2}\right) \underbrace{(1-\operatorname{Erf}(z))}_{\equiv \operatorname{Erfc}(z)}=\sum_{m=0}^{\infty} \frac{z^{2 m}}{m !}-\sum_{m=0}^{\infty} \frac{z^{2 m+1}}{\left(m+\frac{1}{2}\right) !},
$$

where the Error function “ $\operatorname{Erf}(z)$ " is defined by ${ }^{5}$

$$
\operatorname{Erf}(z)=\frac{2}{\sqrt{\pi}} \int_{0}^{z} \mathrm{~d} u \exp \left(-u^{2}\right)=\frac{2}{\sqrt{\pi}} z_{1} \mathrm{~F}_{1}\left(\frac{1}{2} ; \frac{3}{2} ;-z^{2}\right)=-\operatorname{Erf}(-z),
$$

and $\operatorname{Erfc}(z) \equiv 1-\operatorname{Erf}(z)$ is the Complementary error function and, furthermore, ${ }_{1} \mathrm{~F}_{1}(a ; c ; u)$ the Confluent Hypergeometric function. The Taylor series of both of the symmetric and of the antisymmetric parts of $\exp \left(z^{2}\right) \operatorname{Erfc}(z)$ are well known.

In the next two examples we use the Generating function for Hermite polynomials $\mathrm{H}_{n}(z)$

$$
\begin{aligned}
& f(z ; t) \equiv \sum_{n=0}^{\infty} \frac{\mathrm{H}_{n}(z)}{n !} t^{n}=\exp \left(2 z t-t^{2}\right), \\
& f^{(n)}(z ; 0) \equiv\left\{\frac{\partial^{n}}{\partial t^{n}} f(z ; t)\right\}_{t=0}=\mathrm{H}_{n}(z),
\end{aligned}
$$

as starting point for our modified Sumudu or Gauss transformations according to (5.15) and (5.16). Their special cases for even and odd Hermite polynomials are immediately to obtain from (6.5) by separating the symmetrical and the antisymmetrical part with respect to variable $z$

$$
\begin{gathered}
\sum_{m=0}^{\infty} \frac{\mathrm{H}_{2 m}(z)}{(2 m) !} t^{2 m}=\operatorname{ch}(2 z t) \exp \left(-t^{2}\right), \\
\sum_{m=0}^{\infty} \frac{\mathrm{H}_{2 m+1}(z)}{(2 m+1) !} t^{2 m+1}=\operatorname{sh}(2 z t) \exp \left(-t^{2}\right),
\end{gathered}
$$

where the variable $z$ is a parameter. From (5.15) we find then

$$
\begin{aligned}
\hat{f}_{+}^{\frac{1}{2}}(z ; x) \equiv & \sum_{m=0}^{\infty} \frac{\mathrm{H}_{2 m}(z)}{m !}\left(\frac{x}{2}\right)^{2 m} \\
= & \frac{2}{\sqrt{\pi x^{2}}} \int_{0}^{\infty} \mathrm{d} y \operatorname{ch}(2 z y) \exp \left(-y^{2}\right) \exp \left(-\frac{y^{2}}{x^{2}}\right) \\
= & \frac{1}{\sqrt{\pi x^{2}}} \exp \left(\frac{z^{2} x^{2}}{1+x^{2}}\right) \cdot \int_{0}^{\infty} \mathrm{d} y\left\{\exp \left(-\frac{1+x^{2}}{x^{2}}\left(y-\frac{z x^{2}}{1+x^{2}}\right)^{2}\right)\right. \\
& \left.+\exp \left(-\frac{1+x^{2}}{x^{2}}\left(y+\frac{z x^{2}}{1+x^{2}}\right)^{2}\right)\right\}
\end{aligned}
$$

and from (5.16)

${ }^{5}$ In older sources (e.g., [13], chap. 9.9.) the Error function is defined under the same notation without the factor $\frac{2}{\sqrt{\pi}} \approx 1.12838$ but in the modern definition (6.4) with the consequence $\operatorname{Erf}( \pm \infty)= \pm 1$ it is programmed in "Mathematica" (now mostly denoted "erf(z)"). 


$$
\begin{aligned}
\hat{f}_{-}^{\frac{1}{2}}(z ; x) \equiv & \sum_{m=0}^{\infty} \frac{\mathrm{H}_{2 m+1}(z)}{\left(m+\frac{1}{2}\right) !}\left(\frac{x}{2}\right)^{2 m+1} \\
= & \frac{2}{\sqrt{\pi x^{2}}} \int_{0}^{\infty} \mathrm{d} y \operatorname{sh}(2 z y) \exp \left(-y^{2}\right) \exp \left(-\frac{y^{2}}{x^{2}}\right) \\
= & \frac{1}{\sqrt{\pi x^{2}}} \exp \left(\frac{z^{2} x^{2}}{1+x^{2}}\right) \cdot \int_{0}^{\infty} \mathrm{d} y\left\{\exp \left(-\frac{1+x^{2}}{x^{2}}\left(y-\frac{z x^{2}}{1+x^{2}}\right)^{2}\right)\right. \\
& \left.-\exp \left(-\frac{1+x^{2}}{x^{2}}\left(y+\frac{z x^{2}}{1+x^{2}}\right)^{2}\right)\right\} .
\end{aligned}
$$

The integrals in (6.7) and in (6.8) can be evaluated (see Appendix A). From (A2) follows with corresponding substitutions the simple result

$$
\hat{f}_{+}^{\frac{1}{2}}(z ; x)=\sum_{m=0}^{\infty} \frac{\mathrm{H}_{2 m}(z)}{m !}\left(\frac{x}{2}\right)^{2 m}=\frac{1}{\sqrt{1+x^{2}}} \exp \left(\frac{z^{2} x^{2}}{1+x^{2}}\right),
$$

and from $(\mathrm{A} 3)$

$$
\hat{f}_{-}^{\frac{1}{2}}(z ; x)=\sum_{m=0}^{\infty} \frac{\mathrm{H}_{2 m+1}(z)}{\left(m+\frac{1}{2}\right) !}\left(\frac{x}{2}\right)^{2 m+1}=\frac{1}{\sqrt{1+x^{2}}} \exp \left(\frac{z^{2} x^{2}}{1+x^{2}}\right) \operatorname{Erf}\left(\frac{z x}{\sqrt{1+x^{2}}}\right)
$$

With the substitution $x=\mathrm{i} t$ one obtains from (6.9)

$$
\sum_{m=0}^{\infty} \frac{(-1)^{m} \mathrm{H}_{2 m}(z)}{m !}\left(\frac{t}{2}\right)^{2 m}=\frac{1}{\sqrt{1-t^{2}}} \exp \left(-\frac{z^{2} t^{2}}{1-t^{2}}\right),
$$

and from (6.10) with definition of the Error function of imaginary argument $\operatorname{Erfi}(u)$

$$
\begin{aligned}
& \sum_{m=0}^{\infty} \frac{(-1)^{m} \mathrm{H}_{2 m+1}(z)}{\left(m+\frac{1}{2}\right) !}\left(\frac{t}{2}\right)^{2 m+1} \\
& =\frac{1}{\sqrt{1-t^{2}}} \exp \left(-\frac{z^{2} t^{2}}{1-t^{2}}\right)(\mp \mathrm{i}) \operatorname{Erf}\left( \pm \mathrm{i} \frac{z t}{\sqrt{1-t^{2}}}\right) \\
& \equiv \frac{1}{\sqrt{1-t^{2}}} \exp \left(-\frac{z^{2} t^{2}}{1-t^{2}}\right) \operatorname{Erfi}\left(\frac{z t}{\sqrt{1-t^{2}}}\right) .
\end{aligned}
$$

The generating function (6.11) can also be obtained from the following generating function for the product of two Hermite polynomials (formula of Mehler, [13], chap. 10.13., Eq. (22); see also [14])

$$
\sum_{n=0}^{\infty} \frac{\mathrm{H}_{n}(x) \mathrm{H}_{n}(y)}{n !}\left(\frac{t}{2}\right)^{n}=\frac{1}{\sqrt{1-t^{2}}} \exp \left(\frac{2 x y t-\left(x^{2}+y^{2}\right) t^{2}}{1-t^{2}}\right),
$$

by setting $y=0$ and using

$$
\mathrm{H}_{2 m}(0)=\frac{(-1)^{m}(2 m) !}{m !}, \quad \mathrm{H}_{2 m+1}(0)=0 .
$$


It seems that the generating function (6.10) or equivalently (6.12) is new and is not easily to obtain by other methods (e.g., integration of (6.13) with respect to variable $y$ and limiting transition).

We mention that the Sumudu transformation of the basic generating function (6.5) for Hermite polynomials according to (1.2) to a function

$$
\hat{f}(z ; t) \equiv \sum_{n=0}^{\infty} \mathrm{H}_{n}(z) t^{n}
$$

with parameter $z$ leads to a divergent series which turns out to be an asymptotic series of a function which can be evaluated. We make a few remarks about this in next Section. However, one may pose the problem of a transformation also in opposite direction that means to evaluate the series $\sum_{n=0}^{\infty} \frac{\mathrm{H}_{n}(z)}{n !^{2}} t^{n}$ using (6.5) that is equivalent to an inverse Sumudu transformation. This we deal with in the Section after the next.

\section{Sumudu Transformation of Hermite Polynomials and of Their Basic Generating Function}

Since Sumudu transformations transforms powers $z^{n}$ into $n ! z^{n}$ the Sumudu transformation of Hermite polynomials transforms them according to their explicit representation as follows

$$
f(z)=\mathrm{H}_{n}(z) \stackrel{\text { Sumudu }}{\longrightarrow} \hat{f}(z)=n ! \sum_{k=0}^{\left[\frac{n}{2}\right]} \frac{(-1)^{k}}{k !}(2 z)^{n-2 k}
$$

Such transformations may easily be written down in similar way for all other explicitly given polynomials. As a rule, such truncated series are not introduced as Special functions with a special function symbol and, clearly, problems of their convergence also do not exist. In contrast, the case of infinite series, in particular, the Sumudu transformation of generating functions can make problems.

We now consider the Sumudu transformation of the basic Generating function for Hermite polynomials

$$
\begin{aligned}
f(z ; t) & =\sum_{n=0}^{\infty} \mathrm{H}_{n}(z) \frac{t^{n}}{n !}=\exp \left(-\frac{1}{4} \frac{\partial^{2}}{\partial z^{2}}\right) \sum_{n=0}^{\infty} \frac{(2 z t)^{n}}{n !} \\
& =\exp \left(-\frac{1}{4} \frac{\partial^{2}}{\partial z^{2}}\right) \exp (2 z t)=\exp \left(2 z t-t^{2}\right),
\end{aligned}
$$

with respect to the variable $t$ and with $z$ as a (in general, complex) parameter. In Appendix B we develop an operational technique to work with the operator $\exp \left(-\frac{1}{4} \frac{\partial^{2}}{\partial z^{2}}\right)$ in combination with exponential functions. If we use the representation (1.12) of the Sumudu transformation of the Generating function of Hermite polynomials with respect to variable $t$ that means to

$$
\hat{f}(z ; t)=\sum_{n=0}^{\infty} \mathrm{H}_{n}(z) t^{n}
$$


we have to look for the interpretation of the following function $\hat{f}(z ; t)$,

$$
\begin{aligned}
\hat{f}(z ; t) & =\Gamma\left(\frac{\partial}{\partial t} t\right) f(z ; t)=\Gamma\left(\frac{\partial}{\partial t} t\right) \exp \left(-\frac{1}{4} \frac{\partial^{2}}{\partial z^{2}}\right) \sum_{n=0}^{\infty} \frac{(2 z t)^{n}}{n !} \\
& =\exp \left(-\frac{1}{4} \frac{\partial^{2}}{\partial z^{2}}\right) \sum_{n=0}^{\infty} \Gamma\left(\frac{\partial}{\partial t} t\right) \frac{(2 z t)^{n}}{n !} \\
& =\exp \left(-\frac{1}{4} \frac{\partial^{2}}{\partial z^{2}}\right) \sum_{n=0}^{+\infty}(2 z t)^{n}=\exp \left(-\frac{1}{4} \frac{\partial^{2}}{\partial z^{2}}\right) \frac{1}{1-2 z t} .
\end{aligned}
$$

whereas the commutativity of the two integral operators $\Gamma\left(\frac{\partial}{\partial t} t\right)$ and $\exp \left(-\frac{1}{4} \frac{\partial^{2}}{\partial z^{2}}\right)$ is fully obvious the change of the order of summation and of the application of the two integral operators is afflicted with some problems because the arising series are no more absolutely convergent. We assume in the following that changing of these operations is possible and that the arising conditionally convergent series can be interpreted after the evaluation as some generalized functions but do not discuss the last in detail. If we apply the Taylor series of the operator $\exp \left(-\frac{1}{4} \frac{\partial^{2}}{\partial z^{2}}\right)$ to the function $\frac{1}{1-2 z t}$ we obtain

$$
\hat{f}(z ; t)=\sum_{m=0}^{\infty} \frac{(-1)^{m}}{m ! 2^{2 m}} \frac{\partial^{2 m}}{\partial z^{2 m}} \frac{1}{1-2 z t}=\frac{1}{1-2 z t} \sum_{m=0}^{\infty} \frac{(-1)^{m}(2 m) !}{m !}\left(\frac{t}{1-2 z t}\right)^{2 m} .
$$

It is obvious that this sum is divergent for arbitrary $t \neq 0$ since from a certain $m$ on the factor $\frac{(2 m) !}{m !}$ grows faster than $\left(\frac{t}{1-2 z t}\right)^{2}$ which last is the quotient of two neighbored terms $\left(\frac{t}{1-2 z t}\right)^{2(m+1)}$ and $\left(\frac{t}{1-2 z t}\right)^{2 m}$. On the other side if we directly make the Sumudu transformation of the Generating function $\exp \left(2 z t-t^{2}\right)$ using (1.9) we find

$$
\begin{aligned}
\hat{f}(z ; t) & =\int_{0}^{+\infty} \mathrm{d} u \exp \left(2 z t u-(t u)^{2}\right) \mathrm{e}^{-u} \\
& =\exp \left\{\left(\frac{2 z t-1}{2 t}\right)^{2}\right\} \int_{0}^{+\infty} \mathrm{d} u \exp \left\{-t^{2}\left(u-\frac{2 z t-1}{2 t^{2}}\right)^{2}\right\} \\
& =\exp \left\{\left(\frac{2 z t-1}{2 t}\right)^{2}\right\} \frac{\sqrt{\pi}}{2|t|}\left\{1+\operatorname{Erf}\left(\frac{2 z t-1}{2|t|}\right)\right\} .
\end{aligned}
$$

This can be considered as the Sumudu transform of the basic Generating function of Hermite polynomials and (7.5) is its asymptotic expansion.

Since the considered new Generating function for Hermite polynomials as mentioned possesses problems of convergence one may also view onto these functions as generalized functions with weak convergence that means as linear continuous functionals over an appropriate space of basis functions. The Geometric series $\sum_{n=0}^{\infty} w^{n}=\frac{1}{1-w}$ (in our case $\left.w=2 z t\right)$ is not convergent for $|w|>1$ 
and its sum is singular at $w=1$. Within the theory of generalized functions one has to determine this singularity more precisely, for example, as principal value $P \frac{1}{1-w}$ or as $\frac{1}{1-w \pm \mathrm{i} 0}$ and in correspondence with this their transforms. We do not try to discuss this more exactly.

\section{A New Kind of Generating Function for Hermite Polynomials by Inversion of the Sumudu Transformation}

In last Section we saw that the Sumudu transformation of the basic Generating function for Hermite polynomials $\sum_{n=0}^{\infty} \mathrm{H}_{n}(z) \frac{t^{n}}{n !}$ leads to the series $\sum_{n=0}^{\infty} \mathrm{H}_{n}(z) t^{n}$ which is not convergent for arbitrary $t \neq 0$. We also saw that this must not necessarily mean that we cannot give it a sense, for example, as some generalized function since it is formally equal to applying the integral operator $\exp \left(-\frac{1}{4} \frac{\partial^{2}}{\partial z^{2}}\right)$ to the geometric series $\sum_{n=0}^{\infty}(2 z t)^{n}=\frac{1}{1-2 z t}$ changing the order of summation and application of the integral operator.

Let us now assume that we want to evaluate the following series

$$
f(z ; t) \equiv \sum_{n=0}^{\infty} \frac{\mathrm{H}_{n}(z)}{n !^{2}} t^{n},
$$

where $\mathrm{H}_{n}(z)$ are the Hermite polynomials and $z$ is a parameter and where $t$ is the variable involved in the transformations. This is equivalent to an inverse Sumudu transformation of the basic generating function (6.5) for Hermite polynomials which we write now

$$
\hat{f}(z ; t) \equiv \sum_{n=0}^{\infty} \frac{\mathrm{H}_{n}(z)}{n !} t^{n}=\exp \left(2 z t-t^{2}\right),
$$

if we consider $\hat{f}(z ; t)$ as Sumudu transform of $f(z ; t)$ in (8.1). However, we try to calculate $f(z ; t)$ in (8.1) directly from its definition. We find here some relations between Hermite polynomials and series of them to categories of Bessel functions with integer indices and also to Laguerre polynomials.

If we use the following alternative definition of Hermite polynomials (e.g., [14])

$$
\mathrm{H}_{n}(z) \equiv \exp \left(-\frac{1}{4} \frac{\partial^{2}}{\partial z^{2}}\right)(2 z)^{n}
$$

which generates the Hermite polynomials $\mathrm{H}_{n}(z)$ by an integral transformation of the power functions $(2 z)^{n}$ and can be represented as a convolution (integration path in $w$-plane widely deformable) we quickly arrive at

$$
\sum_{n=0}^{\infty} \frac{\mathrm{H}_{n}(z)}{n !^{2}} t^{n}=\exp \left(-\frac{1}{4} \frac{\partial^{2}}{\partial z^{2}}\right) \sum_{n=0}^{\infty} \frac{(2 z t)^{n}}{n !^{2}}=\exp \left(-\frac{1}{4} \frac{\partial^{2}}{\partial z^{2}}\right) \mathrm{I}_{0}(2 \sqrt{2 z t}),
$$

where $\mathrm{I}_{0}(u)$ is a standard Bessel function. If we use the Taylor series of the 
integral operator $\exp \left(-\frac{1}{4} \frac{\partial^{2}}{\partial z^{2}}\right)$ we may transform (8.4) according to

$$
\begin{aligned}
\sum_{n=0}^{\infty} \frac{\mathrm{H}_{n}(z)}{n !^{2}} t^{n} & =\sum_{m=0}^{\infty} \frac{(-1)^{m} t^{2 m}}{m !}\left\{\frac{\partial^{2 m}}{\partial u^{2 m}} \mathrm{I}_{0}(2 \sqrt{u})\right\}_{u=2 z t} \\
& =\sum_{m=0}^{\infty} \frac{(-1)^{m} t^{2 m}}{m !} \frac{\mathrm{I}_{2 m}(2 \sqrt{2 z t})}{(\sqrt{2 z t})^{2 m}} .
\end{aligned}
$$

A representation in operator form which is equivalent to the right-hand side of (8.5) can be directly obtained using the Rodrigues definition of Hermite polynomials as follows

$$
\begin{aligned}
\sum_{n=0}^{\infty} \frac{\mathrm{H}_{n}(z)}{n !^{2}} t^{n} & =\exp \left(z^{2}\right) \sum_{n=0}^{\infty} \frac{t^{n}}{n !^{2}}\left(-\frac{\partial}{\partial z}\right)^{n} \exp \left(-z^{2}\right) \\
& =\sum_{n=0}^{\infty} \frac{t^{n}}{n !^{2}}\left(2 z-\frac{\partial}{\partial z}\right)^{n} 1=\mathrm{I}_{0}\left(2 \sqrt{t\left(2 z-\frac{\partial}{\partial z}\right)}\right) 1,
\end{aligned}
$$

where the last line means the application of an operator function $f(z)=1$. We mention in this connection also the following disentanglement formula for the operators $\left(2 z-\frac{\partial}{\partial z}\right)^{n}$ which is an operator identity

$$
\left(2 z-\frac{\partial}{\partial z}\right)^{n}=\sum_{k=0}^{n} \frac{(-1)^{k} n !}{k !(n-k) !} \mathrm{H}_{n-k}(z) \frac{\partial^{k}}{\partial z^{k}} .
$$

Applied to $f(z)=1$ it leads back from the right-hand side of (8.6) to its left-hand side.

In the special case $z=0$ one finds from (8.1)

$$
\begin{aligned}
\sum_{n=0}^{\infty} \frac{\mathrm{H}_{n}(0)}{n !^{2}} t^{n} & =\sum_{m=0}^{\infty} \frac{(-1)^{m} t^{2 m}}{m !(2 m) !}=\sqrt{\pi} \sum_{m=0}^{\infty} \frac{1}{m !^{2}\left(m-\frac{1}{2}\right) !}\left(-\frac{t^{2}}{4}\right)^{m} \\
& ={ }_{0} \mathrm{~F}_{2}\left(-; 1, \frac{1}{2} ;-\frac{t^{2}}{4}\right) .
\end{aligned}
$$

Apart from a special case of the rarely used Hypergeometric Function ${ }_{0} \mathrm{~F}_{2}\left(-; c_{1}, c_{2} ; u\right)$ the right-hand side does not belong to the Special Functions for which an evaluation in closed form is introduced and investigated in literature. This means that one cannot expect a closed representation for the more general series on the right-hand sides in (8.4) or (8.5). Using the formula (1.17) for the inverse Sumudu transformation and the generating function (8.2) for Hermite polynomials one obtains the following integral representation of the function $f(z ; t)$ in $(8.1)$

$$
\begin{aligned}
\sum_{n=0}^{\infty} \frac{\mathrm{H}_{n}(z)}{n !^{2}} t^{n} & =\sum_{m=0}^{\infty} \frac{(-1)^{m} t^{2 m}}{m !} \frac{\mathrm{I}_{2 m}(2 \sqrt{2 z t})}{(\sqrt{2 z t})^{2 m}} \\
& =\frac{1}{\mathrm{i} 2 \pi} \int_{c-\mathrm{i} \infty}^{c+\mathrm{i} \infty} \mathrm{d} s \frac{1}{s} \exp \left(2 \frac{z}{s}-\frac{1}{s^{2}}+t s\right) .
\end{aligned}
$$


The closed evaluation of the integral by a known function is apparently unknown and, as discussed, can hardly be expected but, nevertheless, the transformed series are important because their convergence properties are different from that for the original series (8.1) and may lead to different approximations.

We will make now some further consideration to the series on both sides of (8.5). If we start from this function then the basic generating function for Hermite polynomials (8.2) is its Sumudu transform and one may expect that we can derive some identities. According to the Sumudu transformation (1.5) we have here specialized

$$
\begin{aligned}
& \frac{1}{t} \int_{0}^{+\infty} \mathrm{d} y \sum_{m=0}^{\infty} \frac{(-1)^{m} y^{2 m}}{m !} \frac{\mathrm{I}_{2 m}(2 \sqrt{2 z y})}{(\sqrt{2 z y})^{2 m}} \exp \left(-\frac{y}{t}\right) \\
& =\frac{1}{t} \int_{0}^{+\infty} \mathrm{d} y \sum_{n=0}^{\infty} \frac{\mathrm{H}_{n}(z)}{n !^{2}} y^{n} \exp \left(-\frac{y}{t}\right) .
\end{aligned}
$$

If we change on the left-hand side the order of integration and summation we find that we do not have a correspondence alone of each sum term over $m$ on the left-hand side and over $n$ on the right-hand side. Making the substitution of the integration variable $y=t u$ on the left-hand side we obtain

$$
\begin{aligned}
& \sum_{m=0}^{\infty} \frac{(-1)^{m}}{m !} \frac{1}{t} \int_{0}^{+\infty} \mathrm{d} y y^{2 m} \frac{\mathrm{I}_{2 m}(2 \sqrt{2 z y})}{(\sqrt{2 z y})^{2 m}} \exp \left(-\frac{y}{t}\right) \\
& =\sum_{m=0}^{\infty} \frac{(-1)^{m}}{m !} t^{2 m} \underbrace{\frac{1}{(2 z t)^{m}} \int_{0}^{+\infty} \mathrm{d} u \mathrm{I}_{2 m}(2 \sqrt{2 z t} \sqrt{u}) u^{m} \mathrm{e}^{-u}}_{=\mathrm{e}^{2 z t}} \\
& =\mathrm{e}^{2 z t} \sum_{m=0}^{\infty} \frac{(-1)^{m}}{m !} t^{2 m}=\exp \left(2 z t-t^{2}\right) .
\end{aligned}
$$

This transformation separates the underbraced integrals which depend only on the variable combination $2 z t$. However that the integrals

$$
\frac{1}{a^{m}} \int_{0}^{+\infty} \mathrm{d} x \mathrm{I}_{2 m}(2 \sqrt{a x}) x^{m} \mathrm{e}^{-x}=\mathrm{e}^{a},
$$

are also independent on $m$ is not easily seen. Supposing the knowledge of the independently derived identity (8.5) and of the generating function (8.11) for Hermite polynomials one may even conclude onto the given evaluation of the underbraced integral.

Starting from (8.5) one may derive closed representation of the series by an integral which, however, also does not lead to a full evaluation by known functions. We write (8.5) in the following form with $t=\frac{x}{2}$ and apply formula (5.3)

$$
\begin{aligned}
& \sum_{n=0}^{\infty} \frac{\mathrm{H}_{n}(z)}{n !^{2}}\left(\frac{x}{2}\right)^{n} \\
& =\sum_{m=0}^{\infty} \frac{(2 m) !}{m !}\left(\frac{x}{2}\right)^{2 m} \frac{(-1)^{m}}{(2 m) !}\left\{\frac{\partial^{2 m}}{\partial u^{2 m}} \mathrm{I}_{0}(2 \sqrt{u})\right\}_{u=z x}
\end{aligned}
$$




$$
=\frac{2}{\sqrt{\pi x^{2}}} \int_{0}^{+\infty} \mathrm{d} y \exp \left(-\frac{y^{2}}{x^{2}}\right) \sum_{m=0}^{\infty} \frac{(-1)^{m}}{(2 m) !} y^{2 m}\left\{\frac{\partial^{2 m}}{\partial u^{2 m}} \mathrm{I}_{0}(2 \sqrt{u})\right\}_{u=z x} .
$$

If we apply here the Taylor series of an analytic function $f(z)=f(x+\mathrm{i} y)$ and its symmetrization

$$
\frac{1}{2}(f(x+\mathrm{i} y)+f(x-\mathrm{i} y))=\sum_{m=0}^{\infty} \frac{f^{(2 m)}(x)}{(2 m) !}(-1)^{m} y^{2 m} .
$$

We may represent (8.13) in the following integral form

$$
\sum_{n=0}^{\infty} \frac{\mathrm{H}_{n}(z)}{n !^{2}}\left(\frac{x}{2}\right)^{n}=\frac{1}{\sqrt{\pi x^{2}}} \int_{0}^{+\infty} \mathrm{d} y \exp \left(-\frac{y^{2}}{x^{2}}\right)\left(\mathrm{I}_{0}(2 \sqrt{z x+\mathrm{i} y})+\mathrm{I}_{0}(2 \sqrt{z x-\mathrm{i} y})\right) .
$$

Apparently, an evaluation of the integrals on the right-hand side by well-introduced Special functions is unknown.

\section{Representation of the New Generating Function for Hermite Polynomials by Series of Laguerre Polynomials}

We derive now another representation of the function (8.1) and start from the Rodrigues definition of the Hermite polynomials within the series, an approach which becomes useful for large values of $|z|$ and thus for the asymptotic case $z \rightarrow \infty$

$$
\begin{aligned}
\sum_{n=0}^{\infty} \frac{\mathrm{H}_{n}(z)}{n !^{2}} t^{n} & =\exp \left(z^{2}\right) \sum_{n=0}^{\infty} \frac{(-1)^{n}}{n !^{2}} t^{n} \frac{\partial^{n}}{\partial z^{n}} \exp \left(-z^{2}\right) \\
& =\exp \left(z^{2}\right) \sum_{n=0}^{\infty} \frac{(-1)^{n}}{n !^{2}}\left(t \frac{\partial}{\partial z}\right)^{n} \sum_{l=0}^{\infty} \frac{(-1)^{l}}{l !} z^{2 l} \\
& =\exp \left(z^{2}\right) \sum_{n=0}^{\infty} \sum_{l=0}^{\infty} \frac{(-1)^{n+l}(2 l) !}{n !^{2} l !(2 l-n) !} z^{2 l}\left(\frac{t}{z}\right)^{n} .
\end{aligned}
$$

By interchanging the order of summations in the double sum we find

$$
\begin{aligned}
\sum_{n=0}^{\infty} \frac{\mathrm{H}_{n}(z)}{n !^{2}} t^{n} & =\exp \left(z^{2}\right) \sum_{l=0}^{\infty} \frac{(-1)^{l}}{l !} z^{2 k} \sum_{n=0}^{2 l} \frac{(-1)^{n}(2 l) !}{n !^{2}(2 l-n) !}\left(\frac{t}{z}\right)^{n} \\
& =\exp \left(z^{2}\right) \sum_{l=0}^{\infty} \frac{(-1)^{l}}{l !} z^{2 l} \mathrm{~L}_{2 l}\left(\frac{t}{z}\right)
\end{aligned}
$$

where $\mathrm{L}_{n}(u)$ denotes the Laguerre polynomials, explicitly

$$
\mathrm{L}_{n}(u)=\sum_{k=0}^{n} \frac{(-1)^{k} n !}{k !^{2}(n-k) !} u^{k}=1-n u+\frac{n(n-1)}{2 !^{2}} u^{2}-\cdots .
$$

It is well known that the Laguerre polynomials $\mathrm{L}_{n}(u)$ are a special case of the Confluent Hypergeometric function ${ }_{1} \mathrm{~F}_{1}(a ; c ; z)$ defined by

$$
{ }_{1} \mathrm{~F}_{1}(a ; c ; z)=\frac{(c-1) !}{(a-1) !} \sum_{k=0}^{\infty} \frac{(k+a-1) !}{(k+c-1) !} \frac{z^{k}}{k !},
$$

according to (e.g., [13], chap. 10.12., Eq. (14))

$$
\mathrm{L}_{n}(u)={ }_{1} \mathrm{~F}_{1}(-n ; 1 ; u)=\mathrm{e}^{u}{ }_{1} \mathrm{~F}_{1}(n+1 ; 1 ;-u),
$$


where, clearly, ${ }_{1} \mathrm{~F}_{1}(n+1 ; 1 ;-u)$ is not a polynomial but an entire function represented by the series

$$
{ }_{1} \mathrm{~F}_{1}(n+1 ; 1 ;-u)=\frac{1}{n !} \sum_{k=0}^{\infty} \frac{(-1)^{k}(n+k) !}{k !^{2}} u^{k} .
$$

The transformation of the Confluent Hypergeometric function

$$
{ }_{1} \mathrm{~F}_{1}(a ; c ; z)=\mathrm{e}^{z}{ }_{1} \mathrm{~F}_{1}(c-a ; c ;-z),
$$

used in (9.5) in specialized form is known as the Kummer transformation ([15], chap. 6.3.). The Kummer transformation possesses involutory character that means applied to the right-hand side provides anew the left-hand side.

If one uses (9.5) one may represent (9.2) also in the form

$$
\begin{aligned}
\sum_{n=0}^{\infty} \frac{\mathrm{H}_{n}(z)}{n !^{2}} t^{n} & =\exp \left(z^{2}\right) \sum_{l=0}^{\infty} \frac{(-1)^{l}}{l !} z^{2 l}{ }_{1} \mathrm{~F}_{1}\left(-2 l ; 1 ; \frac{t}{z}\right) \\
& =\exp \left(z^{2}+\frac{t}{z}\right) \sum_{l=0}^{\infty} \frac{(-1)^{l}}{l !} z^{2 l}{ }_{1} \mathrm{~F}_{1}\left(2 l+1 ; 1 ;-\frac{t}{z}\right) .
\end{aligned}
$$

Here and equivalently in (9.2) we separated the factor $\exp \left(z^{2}\right)$ in the evaluation of the left-hand sides and obtained in this way essentially different representations of the considered kind of generating functions. However, again we are not able to evaluate this generating function by known Special functions and transformed it only into another form of series with the perspective to make other approximations than before.

\section{Two Further Kinds of Generating Functions for Hermite Polynomials}

In comparison to (8.1) it is interesting to consider two slightly different Generating functions (or series) for Hermite polynomials as follows, first

$$
\sum_{n=0}^{\infty} \frac{\left(-\frac{1}{2}\right) ! \mathrm{H}_{n}(z)}{n !\left(n-\frac{1}{2}\right) !} t^{n}=\sum_{n=0}^{\infty} \frac{\mathrm{H}_{n}(z)}{(2 n) !}(4 t)^{n}, \quad\left(\left(-\frac{1}{2}\right) !=\sqrt{\pi}\right),
$$

and second

$$
\sum_{n=0}^{\infty} \frac{\left(+\frac{1}{2}\right) ! \mathrm{H}_{n}(z)}{n !\left(n+\frac{1}{2}\right) !} t^{n}=\sum_{n=0}^{\infty} \frac{\mathrm{H}_{n}(z)}{(2 n+1) !}(4 t)^{n}, \quad\left(\left(+\frac{1}{2}\right) !=\frac{\sqrt{\pi}}{2}\right) .
$$

Using the alternative definition of Hermite polynomials (8.3) one may transform (10.1) according to

$$
\begin{aligned}
\sum_{n=0}^{\infty} \frac{\mathrm{H}_{n}(z)}{(2 n) !}(4 t)^{n} & =\exp \left(-\frac{1}{4} \frac{\partial^{2}}{\partial z^{2}}\right) \sum_{n=0}^{\infty} \frac{(8 t z)^{n}}{(2 n) !} \\
& =\exp \left(-\frac{1}{4} \frac{\partial^{2}}{\partial z^{2}}\right) \operatorname{ch}(2 \sqrt{2 t z})
\end{aligned}
$$




$$
=\exp \left(-\frac{1}{4} \frac{\partial^{2}}{\partial z^{2}}\right) \sqrt{\pi} \frac{\mathrm{I}_{-\frac{1}{2}}(2 \sqrt{2 t z})}{(\sqrt{2 t z})^{-\frac{1}{2}}},
$$

and (10.2) according to

$$
\begin{aligned}
\sum_{n=0}^{\infty} \frac{\mathrm{H}_{n}(z)}{(2 n+1) !}(4 t)^{n} & =\exp \left(-\frac{1}{4} \frac{\partial^{2}}{\partial z^{2}}\right) \sum_{n=0}^{\infty} \frac{(8 t z)^{n}}{(2 n+1) !} \\
& =\exp \left(-\frac{1}{4} \frac{\partial^{2}}{\partial z^{2}}\right) \frac{\operatorname{sh}(2 \sqrt{2 t z})}{2 \sqrt{2 t z}} \\
& =\exp \left(-\frac{1}{4} \frac{\partial^{2}}{\partial z^{2}}\right) \frac{\sqrt{\pi}}{2} \frac{\mathrm{I}^{+\frac{1}{2}}(2 \sqrt{2 t z})}{(\sqrt{2 t z})^{+\frac{1}{2}}} .
\end{aligned}
$$

In the special case $z=0$ with the special values (6.14) of the Hermite polynomials in this case and using the Hypergeometric function ${ }_{0} \mathrm{~F}_{2}\left(-; c_{1}, c_{2} ; u\right)$ one finds from (10.1)

$$
\begin{aligned}
\sum_{n=0}^{\infty} \frac{\mathrm{H}_{n}(0)}{(2 n) !}(4 t)^{n} & =\sqrt{\pi} \sum_{m=0}^{\infty} \frac{(-1)^{m} t^{2 m}}{m !\left(2 m-\frac{1}{2}\right) !} \\
& =\underbrace{\left(-\frac{1}{4}\right) !\left(-\frac{3}{4}\right) !{ }_{0}}_{=\sqrt{2} \pi} \mathrm{F}_{2}\left(-; \frac{3}{4}, \frac{1}{4} ;-\frac{t^{2}}{4}\right),
\end{aligned}
$$

and from (10.2)

$$
\begin{aligned}
\sum_{n=0}^{\infty} \frac{\mathrm{H}_{n}(0)}{(2 n+1) !}(4 t)^{n} & =\frac{\sqrt{\pi}}{2} \sum_{m=0}^{\infty} \frac{(-1)^{m} t^{2 m}}{m !\left(2 m+\frac{1}{2}\right) !} \\
& =\underbrace{\left(+\frac{1}{4}\right) !\left(-\frac{1}{4}\right) !_{0} \mathrm{~F}_{2}\left(-; \frac{5}{4}, \frac{3}{4} ;-\frac{t^{2}}{4}\right) .}_{=\frac{\pi}{2 \sqrt{2}}}
\end{aligned}
$$

This suggests that one cannot write the evaluation of the general series (10.1) and (10.2) in closed form by well-introduced functions.

Inserting the Rodrigues definition of the Hermite polynomials in (10.1) we obtain by a calculation in full analogy to (9.1) and (9.2)

$$
\begin{aligned}
\sum_{n=0}^{\infty} \frac{\mathrm{H}_{n}(z)}{(2 n) !}(4 t)^{n} & =\exp \left(z^{2}\right) \sum_{l=0}^{\infty} \frac{(-1)^{l}}{l !} z^{2 l} \sqrt{\pi} \frac{(2 l) !}{\left(2 l-\frac{1}{2}\right) !} \mathrm{L}_{2 l}^{-\frac{1}{2}}\left(\frac{t}{z}\right) \\
& =\exp \left(z^{2}\right) \sum_{l=0}^{\infty} \frac{(-1)^{l}}{l !} z^{2 l}{ }_{1} \mathrm{~F}_{1}\left(2 l ; \frac{1}{2} ; \frac{t}{z}\right),
\end{aligned}
$$

and inserting it in (10.2)

$$
\sum_{n=0}^{\infty} \frac{\mathrm{H}_{n}(z)}{(2 n+1) !}(4 t)^{n}=\exp \left(z^{2}\right) \sum_{l=0}^{\infty} \frac{(-1)^{l}}{l !} z^{2 l} \frac{\sqrt{\pi}}{2} \frac{(2 l) !}{\left(2 l-\frac{1}{2}\right) !} \mathrm{L}_{2 l}^{+\frac{1}{2}}\left(\frac{t}{z}\right)
$$




$$
=\exp \left(z^{2}\right) \sum_{l=0}^{\infty} \frac{(-1)^{l}}{l !} z^{2 l}{ }_{1} \mathrm{~F}_{1}\left(2 l ; \frac{3}{2} ; \frac{t}{z}\right)
$$

The relation of the Associated (or Laguerre-Sonin) polynomials $\mathrm{L}_{n}^{\alpha}(u)$ to the Hypergeometric function ${ }_{1} \mathrm{~F}_{1}(a ; c ; u)$ together with the Kummer transformation (9.7) of ${ }_{1} \mathrm{~F}_{1}(a ; c ; u)$ is

$$
\begin{aligned}
\mathrm{L}_{n}^{\alpha}(u) & =\frac{(n+\alpha) !}{n ! \alpha !}{ }_{1} \mathrm{~F}_{1}(-n ; 1+\alpha ; u) \\
& =\frac{(n+\alpha) !}{n ! \alpha !} \mathrm{e}^{u}{ }_{1} \mathrm{~F}_{1}(n+1+\alpha ; 1+\alpha ;-u) .
\end{aligned}
$$

The primary summation is here substituted by another summation with excerption of the factor $\exp \left(z^{2}\right)$ but a full evaluation of the series by well-established functions was not obtained.

Since a full evaluation of the series for the considered Generating functions could not be found we will make a transition to an integral representation in analogy to (8.15). To shorten slightly the notation we make the same substitution $t \equiv \frac{x}{2}$ as in last Section. Then we find by Taylor series expansion of the operator $\exp \left(-\frac{1}{4} \frac{\partial^{2}}{\partial z^{2}}\right)$ from (10.3) at first

$$
\sum_{n=0}^{\infty} \frac{\mathrm{H}_{n}(z)}{(2 n) !}(2 x)^{n}=\sum_{m=0}^{\infty} \frac{(2 m) !}{m !}\left(\frac{x}{2}\right)^{2 m} \frac{(-1)^{m}}{(2 m) !}\left\{\frac{\partial^{2 m}}{\partial u^{2 m}} \operatorname{ch}(2 \sqrt{u})\right\}_{u=z x},
$$

and analogously from (10.4)

$$
\sum_{n=0}^{\infty} \frac{\mathrm{H}_{n}(z)}{(2 n+1) !}(2 x)^{n}=\sum_{m=0}^{\infty} \frac{(2 m) !}{m !}\left(\frac{x}{2}\right)^{2 m} \frac{(-1)^{m}}{(2 m) !}\left\{\frac{\partial^{2 m}}{\partial u^{2 m}} \frac{\operatorname{sh}(2 \sqrt{u})}{2 \sqrt{u}}\right\}_{u=z x} .
$$

Then using (5.3) and (8.14) we obtain the following possible representations from (10.10)

$$
\sum_{n=0}^{\infty} \frac{\mathrm{H}_{n}(z)}{(2 n) !}(2 x)^{n}=\frac{1}{\sqrt{\pi x^{2}}} \int_{0}^{\infty} \mathrm{d} y \exp \left(-\frac{y^{2}}{x^{2}}\right)(\operatorname{ch}(2 \sqrt{z x+\mathrm{i} y})+\operatorname{ch}(2 \sqrt{z x-\mathrm{i} y})),
$$

and from (10.11)

$\sum_{n=0}^{\infty} \frac{\mathrm{H}_{n}(z)}{(2 n+1) !}(2 x)^{n}=\frac{1}{\sqrt{\pi x^{2}}} \int_{0}^{\infty} \mathrm{d} y \exp \left(-\frac{y^{2}}{x^{2}}\right)\left(\frac{\operatorname{sh}(2 \sqrt{z x+\mathrm{i} y})}{2 \sqrt{z x+\mathrm{i} y}}+\frac{\operatorname{sh}(2 \sqrt{z x-\mathrm{i} y})}{2 \sqrt{z x-\mathrm{i} y}}\right)$.

The representation by Bessel functions of semi-integer index can be taken from (10.3) and (10.4) by comparison with (10.12) and (10.13) and it is interesting to compare it with formula (8.15). The different representations of the Generating functions by series and integrals may be taken as starting point for different approximations.

\section{Conclusions}

It was shown that Sumudu transformation of a function $f(x)$ as a relative of 
Laplace transformation can lead to the evaluation of series by integral transformation of known series. In Section 5 we considered a modified Sumudu transformation of $f(x)$ which contains the Gaussian bell function instead of the Exponential function and can also lead to the evaluation of series by transformation of known series. The most striking example of a Sumudu transformation establishes a connection between the Exponential and the Geometric series. The use of Sumudu transformation in connection with fractional integration is often useful. This extends the arsenal of methods for the evaluation of infinite series or sometimes of integrals apart from other possible applications, for example, in the theory of differential equations. Furthermore, we derived a certain limiting transition from fractional integration to Sumudu transformation.

For the illustration of the Sumudu transformation we gave simple basic examples and applied it in Sections $7-10$ to obtain new Generating functions for Hermite polynomials. To each function with known Laplace transformation and known Taylor series one may construct a new series which one may evaluate. It is difficult to predict whether or not such a programme will be realized for the great number of Laplace transformations of functions which one may find in the published tables (e.g., [8] [9] [10]). In Section 6 we examined examples for the considered Gauss transformation leading to new Generating functions for Hermite polynomials. In Section 7 we investigated a Sumudu transformation to a new kind of Generating functions for Hermite polynomials which leads to an asymptotic series. In Sections 8 and 9 we considered a series for a new Generating function for Hermite polynomials which corresponds to an inverse Sumudu transformation of the basic Generating function for Hermite polynomials. However, this leads only to transformations of series without the full evaluation of these series by known Special functions. We hope that these examples will find also applications in physics and other sciences.

Almost a decade ago we independently found a transformation which figured in our records as modified Laplace transformation. However, a few years ago we realized that it exists already under the name Sumudu transformation. Since it is closely related to Laplace transformation and the basic integral (1.3) is well known, likely, it was independently discovered several times.

\section{Conflicts of Interest}

The author declares no conflicts of interest regarding the publication of this paper.

\section{References}

[1] Watugala, G.K. (1993) Sumudu Transform: A New Integral Transform to Solve Differential Equations and Control Engineering Problems. International Journal of Mathematical Education in Science and Technology, 24, 35-43. https://doi.org/10.1080/0020739930240105

[2] Belgacem, F.B.M. and Karabelli, A.A. (2006) Sumudu Transform Fundamental 
Properties Investigations and Applications. Journal of Applied Mathematics and Stochastic Analysis, 2006, Article ID: 91083. https://doi.org/10.1155/JAMSA/2006/91083

[3] Belgacem, F.B.M. (2009) Sumudu Applications to Maxwell's Equations. PIERS ONLINE, 5, 355-360. https://doi.org/10.2529/PIERS090120050621

[4] Alenezi, A.M. and Belgacem, F.B.M. (2014) Sumudu Transform Based Treatment of Krawtchouk Polynomials and Their Integral Transforms. AIP Conference Proceedings, 1637, 1395. https://doi.org/10.1063/1.4907306

[5] Demiray, S.T., Bulut, H. and Belgacem, F.B.-M. (2015) Sumudu Transform Method for Analytical Solutions of Fractional Type Ordinary Differential Equations. Mathematical Problems in Engineering, 10, Article ID: 131690.

[6] Katatbeh, Qu. and Belgacem, F.B.M. (2017) Applications of the Sumudu Transform to Fractional Differential Equations. Nonlinear Studies, 18, 99-112. https://www.researchgate.net/publication/234114475

[7] Srivastava, H.M., Luo, M. and Raina, R.K. (2015) A New Integral Transform and Its Applications. Acta Mathematica Scientia, 35, 1386-1400. https://doi.org/10.1016/S0252-9602(15)30061-8

[8] Doetsch, G. (1967) Anleitung zum praktischen Gebrauch der Laplace-Transformation und der Z-Transformation. 3. Aufl, R. Oldenburg, München.

[9] Bateman, H. and Erdélyi, A. (1954) Tables of Integral Transforms, Vol. I. McGraw-Hill, New York.

[10] Ditkin, V.A. and Prudnikov, A.P. (1965) Spravochnik po operatsionnomy ischisleniyu, Vysshaya shkola, Moskva. [Integral Transforms and Operational Calculus.] Pergamon Press, Oxford.

[11] Brychkov, Yu.A. and Prudnikov, A.P. (1977) Integral Transformations of Generalized Functions. Nauka, Moskva. (In Russian)

[12] Zemanian, A.H. (1987) Generalized Integral Transformations. Dover, New York.

[13] Erdélyi, A. (1974) Higher Transcendental Functions, Vol. 2. Nauka, Moskva.

[14] Wünsche, A. (2015) Generating Functions for Products of Laguerre 2D and Hermite 2D Polynomials. Applied Mathematics, 6, 2142-2168.

https://doi.org/10.4236/am.2015.612188

[15] Bateman, H. and Erdélyi, A. (1954) Tables of Integral Transforms, Vol. II. McGraw-Hill, New York.

[16] Vladimirov, V.S. (1971) Equations of Mathematical Physics. 2nd Edition, Nauka, Moskva.

[17] Oldham, K.B. and Spanier, J. (2002) Fractional Calculus. Dover, Mineola.

[18] Samko, S.G., Kilbas, A.A. and Marichev, O.I. (1987) Integrals and Derivatives of Fractional Order and Some of Their Applications. Nauka I Tekhnika, Minsk.

[19] Kranzer, W. (1989) So interessant ist Mathematik. Deutscher Verlag der Wissenschaften, Berlin.

[20] Hirschmann, I.I. and Widder, D.V. (2005) The Convolution Transform. Dover Publ., Mineola (New York).

[21] Louisell, W.H. (1973) Quantum Statistical Properties of Radiation. John Wiley, New York.

[22] Kirillov, A.A. (1972) Elements of Representation Theory. Nauka, Moskva. 
[23] Messiah, A. (1961) Quantum Mechanics. Vol. 1, North Holland, Amsterdam.

[24] Glauber, R.J. (1963) Coherent and Incoherent States of the Radiation Field. Physical Review, 131, 2766-2788. https://doi.org/10.1103/PhysRev.131.2766 


\section{Appendix A}

\section{Evaluation of Two Related Gaussian Integrals}

For convenience although, in principle, known we calculate here two integrals used in Section 6.

With two parameters $a$ and $u$ we have

$$
\begin{aligned}
& \frac{2}{\sqrt{\pi a^{2}}} \int_{0}^{+\infty} \mathrm{d} x \exp \left(-\frac{x^{2}}{a^{2}}\right)\left\{\begin{array}{l}
\operatorname{ch}(u x) \\
\operatorname{sh}(u x)
\end{array}\right\} \\
& =\exp \left(\frac{a^{2} u^{2}}{4}\right) \frac{1}{\sqrt{\pi a^{2}}} \int_{0}^{+\infty} \mathrm{d} x\left\{\exp \left(-\frac{1}{a^{2}}\left(x-\frac{a^{2}}{2} u\right)^{2}\right) \pm \exp \left(-\frac{1}{a^{2}}\left(x+\frac{a^{2}}{2} u\right)^{2}\right)\right\} \\
& =\exp \left(\frac{a^{2} u^{2}}{4}\right) \frac{1}{\sqrt{\pi}}\left\{\int_{-\frac{a u}{2}}^{+\infty} \mathrm{d} y \pm \int_{+\frac{a u}{2}}^{+\infty} \mathrm{d} y\right\} \exp \left(-y^{2}\right) .
\end{aligned}
$$

From this follows in the upper case

$$
\begin{aligned}
& \frac{2}{\sqrt{\pi a^{2}}} \int_{0}^{+\infty} \mathrm{d} x \exp \left(-\frac{x^{2}}{a^{2}}\right) \operatorname{ch}(u x) \\
& =\exp \left(\frac{a^{2} u^{2}}{4}\right) \frac{1}{\sqrt{\pi}} \int_{-\infty}^{+\infty} \mathrm{d} y \exp \left(-y^{2}\right)=\exp \left(\frac{a^{2} u^{2}}{4}\right),
\end{aligned}
$$

and in the lower case

$$
\begin{aligned}
& \frac{2}{\sqrt{\pi a^{2}}} \int_{0}^{+\infty} \mathrm{d} x \exp \left(-\frac{x^{2}}{a^{2}}\right) \operatorname{sh}(u x) \\
& =\exp \left(\frac{a^{2} u^{2}}{4}\right) \frac{1}{\sqrt{\pi}} \int_{-\frac{a u}{2}}^{+\frac{a u}{2}} \mathrm{~d} y \exp \left(-y^{2}\right)=\exp \left(\frac{a^{2} u^{2}}{4}\right) \operatorname{Erf}\left(\frac{a u}{2}\right) .
\end{aligned}
$$

The calculation of the upper and lower case of the integrals in (A1) have to lead to a symmetric and an antisymmetric function with respect to variable $u$, respectively.

\section{Appendix B}

\section{Application of Two Known General Operator Identities to Hermite Polynomials and Its Basic Generating Function}

For application to the calculation of the basic Generating functions for Hermite polynomials we collect in this Appendix two known operator identities without their proofs which can be found in monographs about group theory and its representation theory and specialize them.

For two arbitrary linear operators $A$ and $B$ in a (in general, infinite-dimensional) linear space the following operator expansion is true (e.g., Louisell [21], p. 136)

$$
\begin{aligned}
\mathrm{e}^{A} B \mathrm{e}^{-A} & =B+\frac{1}{1 !}[A, B]+\frac{1}{2 !}[A,[A, B]]+\cdots \\
& =\sum_{n=0}^{\infty} \frac{1}{n !} \underbrace{[A,[A, \cdots[A, B] \cdots]]}_{n \times},
\end{aligned}
$$

with $[A, B] \equiv A B-B A$ denoting the commutator of $A$ and $B$. 
The second operator identity which we consider here is a special case of the general product of two exponential operators $\mathrm{e}^{A} \mathrm{e}^{B}=\mathrm{e}^{f(A, B)}$ which is dealt with in group theory in the transition from a Lie algebra to its Lie group by exponentiation of the general Lie algebra operators and is the content of the Baker-Campbell-Hausdorff-Dynkin formula (e.g., Kirillov [22], p. 119). The here considered special case is (e.g., Messiah [23], Glauber [24] and Louisell [21])

$$
\mathrm{e}^{A} \mathrm{e}^{B}=\exp \left(A+B+\frac{1}{2}[A, B]\right), \quad(\text { if }[A,[A, B]]=[B,[A, B]]=0)
$$

It is only true if the commutator $[A, B]$ commutes with both the operators $A$ and $B$ that means in case of $[A,[A, B]]=[B,[A, B]]=0$. It can be directly proved (Messiah [23], p. 442; see for this proof also Louisell [21], p. 137) without using the general Baker-Campbell-Hausdorff-Dynkin formula which last is quite complicated.

We apply now the theorem (B1) to the special case $A=\exp \left(-\frac{1}{4} \frac{\partial^{2}}{\partial z^{2}}\right)$ and $B=2 t z$ with $t$ considered as a parameter. For the commutator $\left[\frac{\partial^{2}}{\partial z^{2}}, 2 t z\right]$ we find

$$
\left[\frac{\partial^{2}}{\partial z^{2}}, 2 t z\right]=2 t\left\{\frac{\partial^{2}}{\partial z^{2}} z-z \frac{\partial^{2}}{\partial z^{2}}\right\}=2 t z\left\{\left(\frac{\partial}{\partial z}+\frac{1}{z}\right)^{2}-\frac{\partial^{2}}{\partial z^{2}}\right\}=4 t \frac{\partial}{\partial z} .
$$

From this follows

$$
\left[\frac{\partial^{2}}{\partial z^{2}},\left[\frac{\partial^{2}}{\partial z^{2}}, 2 t z\right]\right]=4 t\left[\frac{\partial^{2}}{\partial z^{2}}, \frac{\partial}{\partial z}\right]=0 .
$$

Therefore, the series in (B1) truncates after the sum term to $n=1$ and we find

$$
\exp \left(-\frac{1}{4} \frac{\partial^{2}}{\partial z^{2}}\right)(2 t z) \exp \left(\frac{1}{4} \frac{\partial^{2}}{\partial z^{2}}\right)=t\left(2 z-\frac{\partial}{\partial z}\right)
$$

From this follows

$$
\begin{aligned}
& \exp \left(-\frac{1}{4} \frac{\partial^{2}}{\partial z^{2}}\right) \exp (2 t z) \exp \left(\frac{1}{4} \frac{\partial^{2}}{\partial z^{2}}\right) \\
& =\exp \left\{t\left(2 z-\frac{\partial}{\partial z}\right)\right\}=\exp \left(2 t z-t^{2}\right) \exp \left(-t \frac{\partial}{\partial z}\right)
\end{aligned}
$$

and, more generally, for an arbitrary (smooth) function $f(z)$

$$
\exp \left(-\frac{1}{4} \frac{\partial^{2}}{\partial z^{2}}\right) f(z) \exp \left(\frac{1}{4} \frac{\partial^{2}}{\partial z^{2}}\right)=f\left(z-\frac{1}{2} \frac{\partial}{\partial z}\right)
$$

but only in few cases one may easily disentangle the operator function $f\left(z-\frac{1}{2} \frac{\partial}{\partial z}\right)$ on the right-hand side (i.e. separate into products of functions of $z$ and of $\frac{\partial}{\partial z}$ ). The operator disentanglement on the right-hand side of (B6) in case of the ex- 
ponential function is true according to the theorem (B2) from which we conclude in special case

$$
\begin{aligned}
& \exp (2 t z) \exp \left(-t \frac{\partial}{\partial z}\right) \\
& =\exp \left\{t\left(2 z-\frac{\partial}{\partial z}\right)-\frac{1}{2} 2 t^{2}\left[z, \frac{\partial}{\partial z}\right]\right\} \\
& =\exp \left\{t\left(2 z-\frac{\partial}{\partial z}\right)\right\} \exp \left(t^{2}\right), \quad\left(\left[z, \frac{\partial}{\partial z}\right]=-1\right),
\end{aligned}
$$

since the commutator $\left[z, \frac{\partial}{\partial z}\right]=-1$ commutes with the operators $z$ and $\frac{\partial}{\partial z}$.

On the right-hand side of the operator identity (B6) we have separated the basic Generating function for Hermite polynomials $\sum_{n=0}^{\infty} \mathrm{H}_{n}(z) \frac{t^{n}}{n !}=\exp \left(2 z t-t^{2}\right)$. This operator identity can be represented equivalently

$$
\exp \left(-\frac{1}{4} \frac{\partial^{2}}{\partial z^{2}}\right) \exp (2 t z)=\exp \left(2 z t-t^{2}\right) \exp \left(-t \frac{\partial}{\partial z}-\frac{1}{4} \frac{\partial^{2}}{\partial z^{2}}\right)
$$

On this occasion we mention that from (B5) with $t=1$ follows the operator identity

$$
\begin{aligned}
\exp \left(-\frac{1}{4} \frac{\partial^{2}}{\partial z^{2}}\right)(2 z)^{n} & =\left(2 z-\frac{\partial}{\partial z}\right)^{n} \exp \left(-\frac{1}{4} \frac{\partial^{2}}{\partial z^{2}}\right) \\
& =\sum_{k=0}^{n} \frac{(-1)^{k} n !}{k !(n-k) !} \mathrm{H}_{n-k}(z) \frac{\partial^{k}}{\partial z^{k}} \exp \left(-\frac{1}{4} \frac{\partial^{2}}{\partial z^{2}}\right)
\end{aligned}
$$

where in addition we used the operator identity (8.7). Applied to the function $f(z)=1$ it generates the Hermite polynomials $\mathrm{H}_{n}(z)$ as we repeatedly used it. For an arbitrary function $f(z)$ with Taylor series at $z=0$ follows from (B10) by reordering of the sum terms in the arising double sum the operator identity

$$
\begin{aligned}
f\left(z-\frac{1}{2} \frac{\partial}{\partial z}\right) & =\sum_{n=0}^{\infty} \frac{f^{(n)}(0)}{n !} \frac{1}{2^{n}} \sum_{k=0}^{n} \frac{(-1)^{k} n !}{k !(n-k) !} \mathrm{H}_{n-k}(z) \frac{\partial^{k}}{\partial z^{k}} \\
& =\sum_{k=0}^{\infty} \frac{(-1)^{k}}{k ! 2^{k}}\left(\sum_{l=0}^{\infty} \frac{f^{(k+l)}(0)}{l ! 2^{l}} \mathrm{H}_{l}(z)\right) \frac{\partial^{k}}{\partial z^{k}} .
\end{aligned}
$$

The inner sum is of the form of Generating functions for Hermite polynomials (without powers of a parameter) and cannot be evaluated in closed way in general case. 